\title{
Tensor networks demonstrate the robustness of localization and symmetry-protected topological phases
}

\author{
Thorsten B. Wahl \\ Rudolf Peierls Centre for Theoretical Physics, Clarendon Laboratory, Parks Road, Oxford OX1 3PU, United Kingdom
}

(Received 8 January 2018; revised manuscript received 20 July 2018; published 14 August 2018)

\begin{abstract}
We prove that all eigenstates of many-body localized symmetry-protected topological systems with time reversal symmetry have fourfold degenerate entanglement spectra in the thermodynamic limit. To that end, we employ unitary quantum circuits where the number of sites the gates act on grows linearly with the system size. We find that the corresponding matrix product operator representation has similar local symmetries as matrix product ground states of symmetry-protected topological phases. Those local symmetries give rise to a $\mathbb{Z}_{2}$ topological index, which is robust against arbitrary perturbations so long as they do not break time reversal symmetry or drive the system out of the fully many-body localized phase.
\end{abstract}

DOI: 10.1103/PhysRevB.98.054204

\section{INTRODUCTION}

The idea that systems out of equilibrium act as their own heat bath was first challenged by Anderson in 1958 [1]. Later works confirmed rigorously that in noninteracting one- and two-dimensional systems (without broken time reversal symmetry or spin-orbit coupling) arbitrarily weak disorder leads to localization of all single particle eigenstates [2]. Strikingly, in one dimension, the resulting lack of transport survives for sufficiently strong disorder if interactions are included [3-6]. Such many-body localized (MBL) systems [7-12] retain a memory of their initial state for arbitrarily long times, thus violating the eigenstate thermalization hypothesis (ETH) [13-19]. Manybody localization was observed in recent optical lattice experiments on one- [20] and two-dimensional systems [21,22]. Numerical studies predict other exotic phenomena in MBL systems, such as the logarithmic growth of entanglement following a quantum quench [23-28] and an unconventional transition to the thermal phase $[8,29-47]$. From a conceptual point of view, MBL systems are characterized by an extensive number of local integrals of motion (LIOM) [10,48-58] and area-law entangled eigenstates [59-61]. Excited eigenstates thus have similar features as the ground states of local gapped Hamiltonians [62], which is why those eigenstates can be efficiently approximated by matrix product states (MPS) [59,6369]. Moreover, unitary quantum circuits (a special type of tensor networks [63,70-73]) encode the entire set of eigenstates efficiently [74-77].

The absence of thermal fluctuations in MBL systems facilitates symmetry-breaking orders and symmetry-protected topological (SPT) orders at all energy scales, which in clean systems can only exist at zero temperature [59,78-83]. Hence, in the localized case all eigenstates can be SPT, which makes MBL systems viable candidates for topological quantum memories at arbitrary energy density [59]. Symmetry and localization protected systems thus interface with quantum information theory both at their theoretical description by tensor networks and their practical potential for quantum information storage and processing tasks $[59,79]$.

One of the greatest accomplishments in tensor network research so far was the classification of all gapped topological phases in one dimension [84-86]. This was made possible by the insight that ground states of one-dimensional gapped systems can be efficiently approximated by MPS $[87,88]$. This suggests that tensor networks might also be used to classify SPT MBL phases as proposed in Ref. [79].

In this article we establish tensor networks as a tool for such a classification. Specifically, we use quantum circuits to prove that MBL phases in one dimension protected by time reversal symmetry fall in two different classes given by a $\mathbb{Z}_{2}$ topological index. The only assumption we make in our proof is that a two-layer unitary quantum circuit [77] diagonalizes the MBL Hamiltonian exactly in the thermodynamic limit if the length of the gates increases linearly with the system size. As we argue, this applies to MBL systems as defined above, also known as fully many-body localized (FMBL) systems [89], which do not possess a mobility edge [36,90]. We find that the global time reversal symmetry of the system gives rise to local symmetries of the tensors-similarly to MPS with a global symmetry $[84,91]$. We also prove that the topological index determined by those local symmetries is robust against arbitrary symmetry respecting perturbations as long as they do not drive the system out of the FMBL phase. Finally, we show that all eigenstates in the SPT MBL phase have fourfold degenerate entanglement spectra.

In the following section we give a very brief introduction into symmetry-protected topological many-body localized phases. Section III provides a summary of the main results and an intuitive (nontechnical) outline of the stability proof, which follows in Sec. IV. Section V concludes the paper and gives an outlook for future work.

Those readers interested only in the general MBL classification idea using tensor networks and the physical implications may skip Sec. IV. 


\section{SYMMETRY AND LOCALIZATION PROTECTED PHASES}

\section{A. Local integrals of motion}

Throughout this article we consider a disordered spins chain in one dimension with periodic boundary conditions. For sufficiently strong disorder, where the system is in the FMBL phase, the Hamiltonian commutes with an extensive number of LIOMs $\tau_{z}^{i},\left[H, \tau_{z}^{i}\right]=\left[\tau_{z}^{i}, \tau_{z}^{j}\right]=0 . \tau_{z}^{i}$ is related to $\sigma_{z}^{i}$ (Pauli- $z$ operator at site $i$ ) by a quasilocal unitary transformation $U$, i.e., $\tau_{z}^{i}=U \sigma_{z}^{i} U^{\dagger}$ is an effective spin exponentially localized around site $i$ [49]. Note that $U$ also diagonalizes the Hamiltonian. The eigenstates can be labeled by the eigenvalues of the $\tau_{z}^{i}$ operators, known as l-bits. The decay length $\xi_{i}$ of $\tau_{z}^{i}$ depends on the specific disorder realization. In the FMBL phase, the likelihood of finding a decay length of order $O(N)$ is zero in the $\operatorname{limit} N \rightarrow \infty[49,56]$.

\section{B. Symmetry and localization protected phases}

In FMBL systems, all eigenstates fulfill the area law of entanglement. This allows, in principle, for the topological symmetry protection of the full set of eigenstates. In one-dimensional systems, time reversal symmetry or on-site symmetries given by an Abelian symmetry group [92] are candidates. (Note that as opposed to ground states of clean systems, for random disordered systems, inversion symmetry is not an option.) In this article we will show the robustness of time reversal symmetry-protected MBL systems and point out what currently prevents the generalization to on-site symmetry groups (see Sec. III).

As a paradigmatic example, consider the disordered cluster model with random couplings [81],

$$
H=\sum_{i=1}^{N}\left(\lambda_{i} \sigma_{x}^{i-1} \sigma_{z}^{i} \sigma_{x}^{i+1}+h_{i} \sigma_{z}^{i}+V_{i} \sigma_{z}^{i} \sigma_{z}^{i+1}\right)
$$

on a chain with $N$ sites and periodic boundary conditions. (We define position indices modulo $N$.) $\lambda_{i}, h_{i}$, and $V_{i}$ are real and chosen independently from a Gaussian distribution with standard deviation $\sigma_{\lambda}, \sigma_{h}$, and $\sigma_{V}$, respectively. In Ref. [81] it was observed numerically that the entanglement spectra of all eigenstates are approximately fourfold degenerate for $\sigma_{h}, \sigma_{V} \ll \sigma_{\lambda}$ and finite $N$. We prove the exact degeneracy in the limit $N \rightarrow \infty$ using the fact that the corresponding SPT MBL phase is protected by time reversal symmetry, which in this case is a combination of complex conjugation $\left(^{*}\right)$ and rotation by $\sigma_{z}$,

$$
H=\sigma_{z}^{\otimes N} H^{*} \sigma_{z}^{\otimes N}
$$

In general, time reversal acts as $\mathcal{T}=K v^{\otimes N}$, where $K$ denotes complex conjugation and $v$ an on-site unitary operation with $v v^{*}= \pm \mathbb{1}$. Note that the sign will not affect the topological classification [85], as the overall unitary $\gamma^{\otimes N}$ fulfills $\gamma^{\otimes N} v^{* \otimes N}=$ $( \pm 1)^{N} \mathbb{1}$, which is $\mathbb{1}$ for even $N$. (If $N$ is odd, one can always add a completely decoupled auxiliary spin to the chain, which would not change the fact that the system is MBL.)

\section{NONTECHNICAL SUMMARY OF RESULTS AND INTUITIVE OUTLINE OF THE PROOF}

Numerical evidence indicates that two-layer quantum circuits with long gates approximate FMBL systems efficiently [77]. For disordered systems, they are thus the fullspectrum analogs of matrix product states (MPS) for clean systems. This suggests that they might also be used for the classification of symmetry-protected MBL systems — as MPS were for clean systems. In this article we provide evidence for this conclusion by using one-dimensional quantum circuits to show that MBL systems protected by time reversal symmetry fall in two different classes, where one of them is topologically nontrivial as exemplified by a fourfold degeneracy of the entanglement spectrum of all of its eigenstates.

The only assumption (other than being in a time reversal symmetric MBL phase) that goes into the proof is that the local integrals of motion can be represented efficiently by a quantum circuit with long gates. This is basically equivalent to not having any LIOM with a decay length of the order of the system size, i.e., to be in the FMBL phase. We show that, as a result, the Hamiltonian belongs to one of two topologically inequivalent phases. We show that it is impossible to connect the two phases adiabatically without violating either the time reversal symmetry or the FMBL condition. This is very reminiscent of SPT ground states of clean Hamiltonians: As long as the symmetry is preserved, they cannot be adiabatically connected to the trivial phase unless they become delocalized (having algebraically decaying correlations), i.e., the gap of the Hamiltonian closes. This is why MPS can be used for their classification: MPS always have exponentially decaying correlations and represent ground states of local gapped Hamiltonians. If the tensors of two (symmetric) MPS cannot be continuously connected, it is impossible to connect the ground states they approximate continuously without encountering a quantum phase transition, at which correlations decay algebraically (which cannot be captured exactly by an MPS).

In the same way, the transition between the two topologically inequivalent MBL phases must lie outside the realm of systems that can be approximated efficiently by quantum circuits. Hence, at the transition, at least one LIOM must become delocalized (which does not imply the transition resembles an MBL-to-thermal transition [80]). This correspondence between MPS classifications of ground states and quantum circuit classifications of MBL phases is summarized in Table I.

The quantum circuits used for the proof are of the form [77]

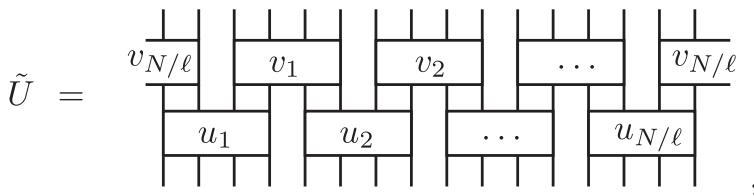

where $u_{k}$ and $v_{k}$ are unitaries (indicated by boxes) acting on $\ell$ sites. Each leg corresponds to a tensor index of dimension 2 , i.e., in the above case $\ell=4$. The lower dangling legs correspond to the approximate 1-bit basis $l_{1}, l_{2}, \ldots, l_{N}$ and the upper open legs to the local physical basis. Connected legs indicate summation over the corresponding indices. $\tilde{U}$ 
TABLE I. Table showing the correspondence between MPS descriptions of ground states and quantum circuit descriptions of the entire set of eigenstates of MBL sytems.

\begin{tabular}{l}
\hline \hline $\begin{array}{l}\text { Property } \\
\text { Description of } \\
\text { System }\end{array}$ \\
$\begin{array}{l}\text { Ansatz } \\
\text { Time reversal symmetry }\end{array}$ \\
$\begin{array}{l}\text { Local symmetry } \\
\text { Consequence }\end{array}$
\end{tabular}

$\overline{\bar{a} \text { MPS can be straightforwardly defined for nontranslationally invariant systems, but using them for a classification of phases in such a case }}$ requires additional tools, such as the renormalization group procedure [85].

approximately diagonalizes the Hamiltonian. The error of the optimized approximation decreases exponentially with $\ell$.

For nondegenerate ground states of clean systems, time reversal symmetry implies

$$
\mathcal{T}|\psi\rangle=\gamma^{\otimes N}\left|\psi^{*}\right\rangle=e^{i \theta}|\psi\rangle
$$

This generalizes to

$$
v^{\otimes N}\left|\psi_{l_{1} \cdots l_{N}}^{*}\right\rangle=e^{i \theta_{l_{1} \cdots l_{N}}}\left|\psi_{l_{1} \cdots l_{N}}\right\rangle
$$

for MBL systems with eigenstates $\left|\psi_{l_{1} \ldots l_{N}}\right\rangle$ and nondegenerate energies (possible energy degeneracies can be removed by adding infinitesimally small perturbations). Since quantum circuits with long gates form an efficient approximation, the same must be true for the approximate eigenstates $\left|\tilde{\psi}_{l_{1} \cdots l_{N}}\right\rangle$ contained in the unitary $\tilde{U}$,

$$
v^{\otimes N}\left|\tilde{\psi}_{l_{1} \cdots l_{N}}^{*}\right\rangle=e^{i \theta_{l_{1} \cdots l_{N}}}\left|\tilde{\psi}_{l_{1} \cdots l_{N}}\right\rangle
$$

For $\tilde{U}$ this implies

$$
\tilde{U} \Theta=\gamma^{\otimes N} \tilde{U}^{*}
$$

where $\Theta$ is the diagonal matrix with elements $e^{i \theta_{l_{1} \cdots l_{N}}} . \Theta^{1 / 2}$ can be absorbed into the two-layer quantum circuit (see Sec. IV for the precise reason for this), i.e., $\tilde{U} \rightarrow \tilde{U} \Theta^{1 / 2}$, such that

$$
\tilde{U}=v^{\otimes N} \tilde{U}^{*}
$$

The absorption of such phase factors only works for time reversal symmetry, which is what currently precludes a generalization to on-site symmetries characterized by a symmetry group $G$. In graphical notation, Eq. (8) reads (we combine groups of $\ell / 2$ lines into single lines with dimension $2^{\ell / 2}$ )
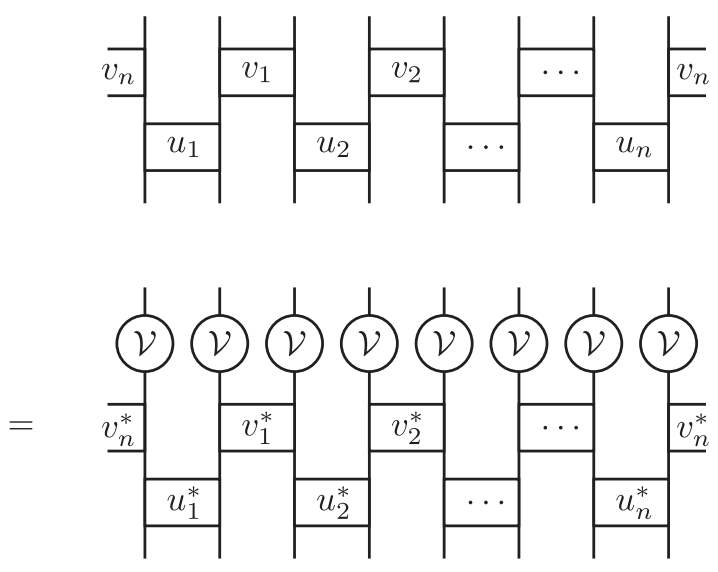

with $\mathcal{V}=v^{\otimes \ell / 2}$. Note that multiplication from left to right in algebraic notation corresponds to top to bottom in graphical notation. If we define $u_{k}^{\prime}=u_{k}^{*}$ and $v_{k}^{\prime}=(\mathcal{V} \otimes \mathcal{V}) v_{k}^{*}$, we discern that Eq. (9) equates two two-layer quantum circuits,
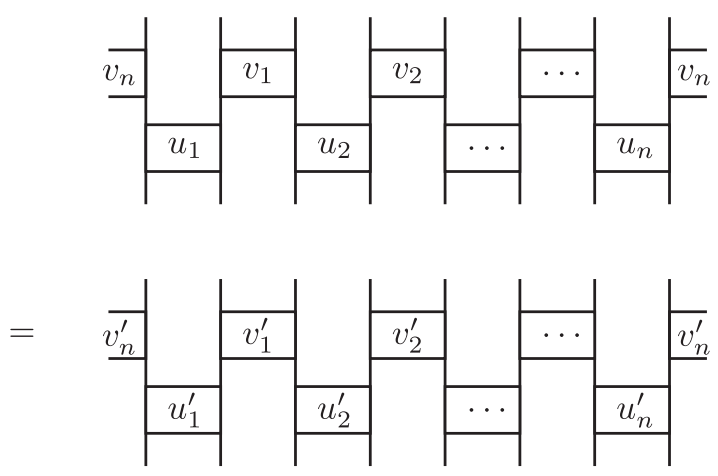
If we multiply both sides from the bottom by $u_{k}^{\prime \dagger}$ for $k=$ $1, \ldots, n$ and from the top by $v_{k}^{\dagger}$, we arrive at

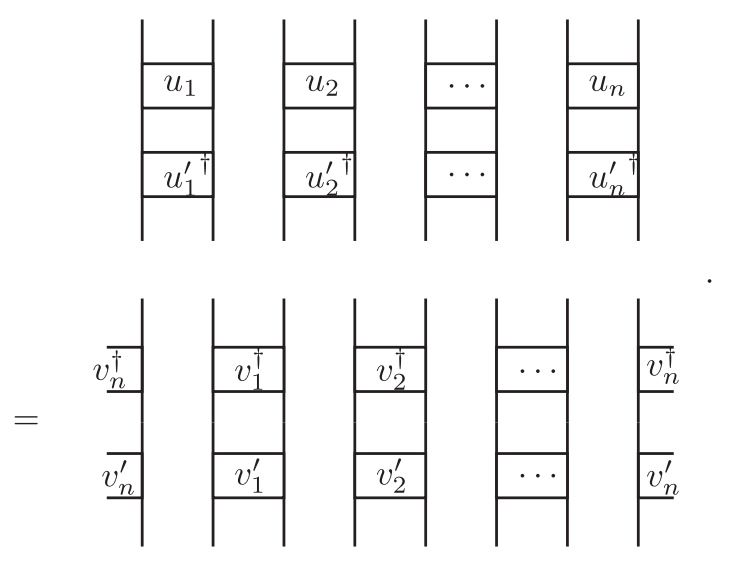

(11)

The left-hand side is a tensor product of $u_{k} u_{k}^{\prime \dagger}$ and the righthand side of $v_{k}^{\dagger} v_{k}^{\prime}$ but shifted by one site with respect to each other. Hence, it has to hold that

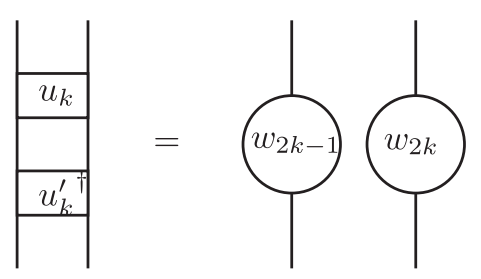

and

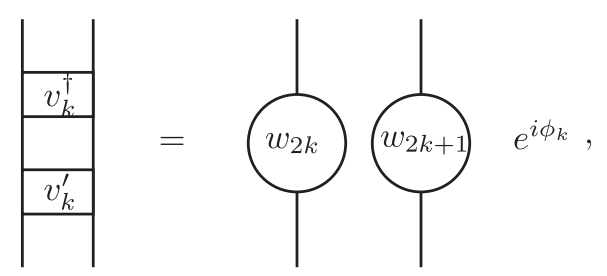

where the $w_{j}$ are unitaries. The phase factor $e^{i \phi_{k}}$ arises because the decomposition of Eq. (11) into a product of tensors acting on blocks of $\frac{\ell}{2}$ sites is unique up to overall factors (which have to be of magnitude 1 due to unitarity). We call Eqs. (12) and (13) a gauge transformation, as it leaves the overall quantum circuit invariant. If we insert back the specific case of $u_{k}^{\prime}=u_{k}^{*}$ and $v_{k}^{\prime}=(\mathcal{V} \otimes \mathcal{V}) v_{k}^{*}$, we obtain

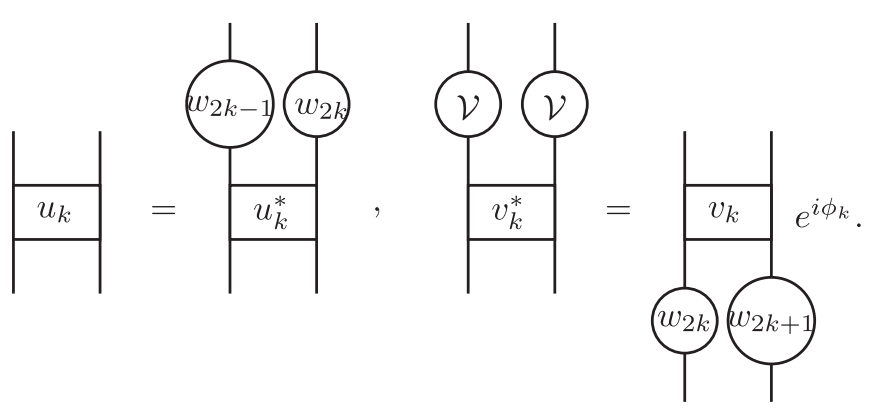

If one takes the complex conjugate of Eqs. (14) and inserts that back into the original Eqs. (14), one obtains

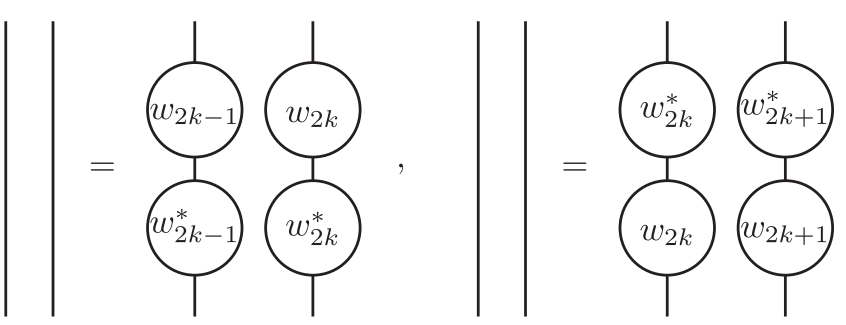

using $\mathcal{V} \mathcal{V}^{*}= \pm \mathbb{1}$. The left equation implies $w_{2 k-1} w_{2 k-1}^{*}=$ $\mathbb{1} e^{i \beta_{k}}, w_{2 k} w_{2 k}^{*}=\mathbb{1} e^{-i \beta_{k}}$ and the right one $w_{2 k} w_{2 k}^{*}=\mathbb{1} e^{i \beta_{k}^{\prime}}$, $w_{2 k+1} w_{2 k+1}^{*}=\mathbb{1} e^{-i \beta_{k}^{\prime}}$. We thus have a single phase $\beta$, $w_{2 k-1} w_{2 k-1}^{*}=\mathbb{1} e^{i \beta}, w_{2 k} w_{2 k}^{*}=\mathbb{1} e^{-i \beta}$, for all $k=1, \ldots, n$. Inserting the resulting $w_{2 k-1}=e^{i \beta} w_{2 k-1}^{\top}$ into itself [84] yields $e^{2 i \beta}=1$, i.e., $w_{j} w_{j}^{*}= \pm \mathbb{1}$ with the same sign for all $j=$ $1,2, \ldots, 2 n$. This is the topological sign of the SPT MBL phase: It does not depend on the site index $k$, i.e., it is the same for the entire chain. One cannot adiabatically change a unitary quantum circuit from a topological index -1 to a +1 index, as continuous variation of the unitaries $\left\{u_{k}, v_{k}\right\}$ corresponds according to Eqs. (14) to continuous variation of $\left\{w_{j}\right\}$, which leaves the sign of $w_{j} w_{j}^{*}= \pm \mathbb{1}$ invariant. This indicates that under adiabatic perturbations of the Hamiltonian, it is impossible to connect the two phases unless the description in terms of local integrals of motion and thus in terms of quantum circuits breaks down. At such a transition point, at least one integral of motion must become delocalized.

Finally, to gain an intuition as to why one of the SPT phases has fourfold degeneracy of all eigenstates, it is illustrative to write $\tilde{U}$ as a matrix product operator (MPO),

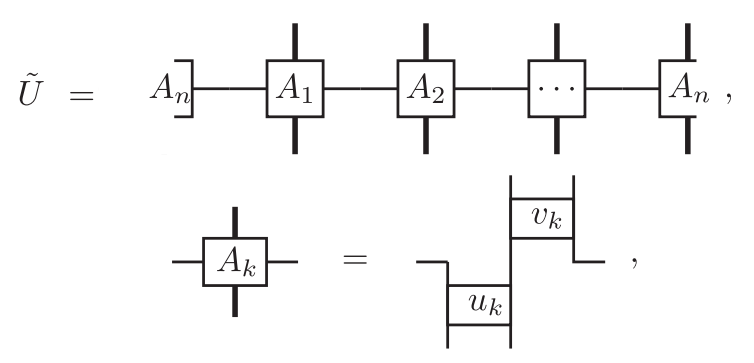

where we use thick lines to denote the combination of two vertical legs to one with dimension $2^{\ell}$. Equation (14) gives

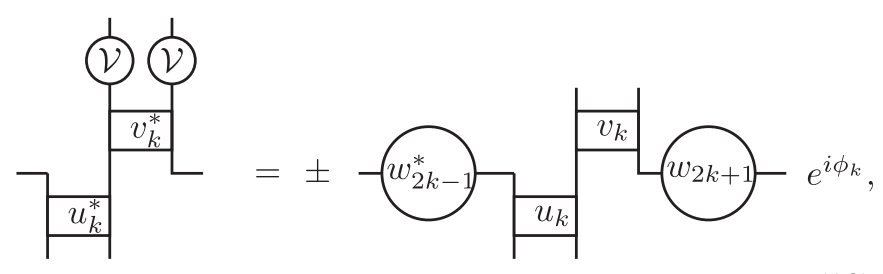


using $w_{2 k}^{*} w_{2 k}= \pm \mathbb{1}$, and therefore

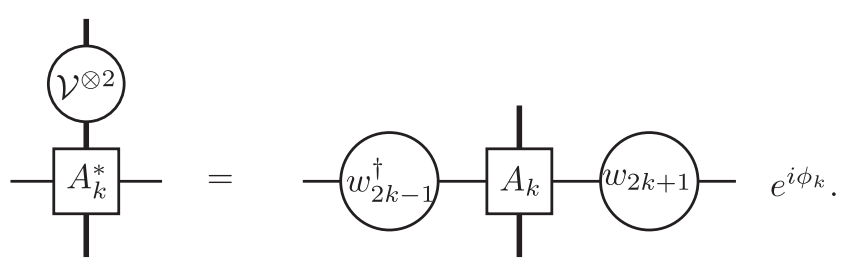

This relation is almost identical to the one obtained for MPS representing time reversal symmetric ground states [84]. The only differences are the lower leg corresponding to the local 1-bit configuration (making it an MPO rather than an MPS) and the breaking of translational invariance reflected by the sitedependent tensors $A_{k}$ and virtual symmetries $w_{2 k-1}, w_{2 k+1}$. However, since $w_{j} w_{j}^{*}= \pm \mathbb{1}$ for all $j$, the same conclusions can be drawn as in Ref. [84]: Consider the case of $w_{j} w_{j}^{*}=-\mathbb{1}$ and a specific eigenstate by fixing the 1-bit configuration, i.e., the indices of the lower legs. The entanglement spectrum of that eigenstate is encoded in a reduced density matrix defined on the virtual space (horizontal legs). Due to Eq. (19), it has to commute with $w_{2 k-1}$ and $w_{2 k+1}$. For $w_{j} w_{j}^{*}=-\mathbb{1}$ this implies that the spectrum of the reduced density matrix has to be fourfold degenerate. Since this conclusion can be drawn independently of the chosen 1-bit configuration, all eigenstates must have fourfold degenerate entanglement spectra.

We thus showed that in the presence of time reversal symmetry, MBL systems fall into one of two topologically distinct phases, which can be distinguished by the entanglement spectra of the individual eigenstates. This is in analogy to the classification of matrix product states with time reversal symmetry [84-86]. Along these lines, we expect a classification by the second cohomology group if the system is invariant under an on-site symmetry given by a certain symmetry group [79]. The technical problems with this extension can be gathered from the following section. Finally, note that the derivations here only apply to bosonic systems; for fermionic systems another symmetry constraint (parity) would have to be imposed [93].

The rigorous demonstration of the results above is the subject of the following section.

\section{THEOREM AND PROOF}

\section{A. Theorem}

If for all sufficiently large $N$ the following conditions are fulfilled

(1) there exists a unitary $U$ diagonalizing the Hamiltonian $H$ defining $\tau_{i}^{z}=U \sigma_{i}^{z} U^{\dagger}$ and a two-layer quantum circuit $\tilde{U}$ with $\tilde{\tau}_{i}^{z}=\tilde{U} \sigma_{i}^{z} \tilde{U}^{\dagger}$ such that $\left\|\tilde{\tau}_{i}^{z}-\tau_{i}^{z}\right\|_{\text {op }}<c e^{-\frac{\ell}{\xi_{i}}}$ with $\xi_{\max }:=$ $\max _{i} \xi_{i}<c^{\prime} N^{1-\mu}$ for some fixed $c, c^{\prime}>0$ and $0<\mu<1$ (efficient approximability)

(2) the Hamiltonian is invariant under time reversal operation $\mathcal{T}=K v^{\otimes N}, H=\mathcal{T} H \mathcal{T}^{\dagger}$ (time reversal symmetry)

(3) conditions 1 and 2 are also fulfilled for the Hamiltonian $H+\epsilon V$ with arbitrary infinitesimally small strictly local perturbations, $\epsilon \rightarrow 0$ (MBL stability),

then the following holds in the thermodynamic limit $(N \rightarrow$ $\infty)$ :

(1) the Hamiltonian belongs to one of two topological classes, where one of them has a full set of eigenstates with fourfold degenerate entanglement spectra (topological property)

(2) under adiabatic perturbations, the Hamiltonian cannot leave its topological class if the above conditions are fulfilled along the path (topological stability).

We will prove each of the two statements in turn.

\section{B. Proof of Statement 1}

We first prove the following:

Lemma 1. Condition 1 of the Theorem implies for $\ell(N)=$ $\alpha N$ to leading order in $N$ that there exists a unitary $U^{\prime}$ exactly diagonalizing the Hamiltonians such that $\left\|U^{\prime}-\tilde{U}\right\|_{\text {op }}<$ $2^{9 / 4} \sqrt{\frac{c N}{3}} e^{-\frac{\alpha N^{\mu}}{2 c^{\prime}}}$.

Proof of Lemma 1. We set $U^{\prime}=U \Phi$, where $\Phi$ (to be specified below) is a diagonal matrix whose nonvanishing elements have magnitude $1 . U^{\prime}$ also diagonalizes the Hamiltonian and has the same LIOMs $\tau_{i}^{z}$. Condition 1 hence implies for $U^{\prime}$

$$
\left\|\sigma_{i}^{z}-\tilde{U}^{\dagger} U^{\prime} \sigma_{i}^{z} U^{\prime \dagger} \tilde{U}\right\|_{\mathrm{op}}<c e^{-\frac{\ell}{\xi_{i}}} .
$$

We write $\tilde{U}^{\dagger} U^{\prime}$ in blocks corresponding to degenerate subspaces of $\sigma_{1}^{z}, \tilde{U}^{\dagger} U^{\prime}:=\left(\begin{array}{ll}U_{11} & U_{12} \\ U_{21} & U_{22}\end{array}\right)$. Then, Eq. (20) results in

$$
\left\|\left(\begin{array}{ll}
U_{11} U_{11}^{\dagger}-U_{12} U_{12}^{\dagger}-\mathbb{1} & U_{11} U_{21}^{\dagger}-U_{12} U_{22}^{\dagger} \\
U_{21} U_{11}^{\dagger}-U_{22} U_{12}^{\dagger} U_{21} & U_{21}^{\dagger}-U_{22} U_{22}^{\dagger}-\mathbb{1}
\end{array}\right)\right\|_{\text {op }}<c e^{-\frac{\ell}{\xi_{i}}}
$$

Since $\tilde{U}^{\dagger} U^{\prime}$ is unitary,

$$
\left(\begin{array}{ll}
U_{11} U_{11}^{\dagger}+U_{12} U_{12}^{\dagger} & U_{11} U_{21}^{\dagger}+U_{12} U_{22}^{\dagger} \\
U_{21} U_{11}^{\dagger}+U_{22} U_{12}^{\dagger} & U_{21} U_{21}^{\dagger}+U_{22} U_{22}^{\dagger}
\end{array}\right)=\left(\begin{array}{cc}
\mathbb{1} & 0 \\
0 & \mathbb{1}
\end{array}\right)
$$

we get

$$
\left\|\left(\begin{array}{cc}
-2 U_{12} U_{12}^{\dagger} & 2 U_{11} U_{21}^{\dagger} \\
-2 U_{22} U_{12}^{\dagger} & 2 U_{21} U_{21}^{\dagger}
\end{array}\right)\right\|_{\text {op }}=2\left\|\left(\begin{array}{cc}
U_{12} & U_{11} \\
U_{22} & U_{21}
\end{array}\right)\left(\begin{array}{cc}
-U_{12}^{\dagger} & 0 \\
0 & U_{21}^{\dagger}
\end{array}\right)\right\|_{\text {op }}=2 \max \left(\left\|U_{12}\right\|_{\text {op }},\left\|U_{21}\right\|_{\text {op }}\right)<c e^{-\frac{\ell}{\xi_{i}}} .
$$


We define $D$ as the diagonal matrix with the same diagonal elements as $\tilde{U}^{\dagger} U^{\prime}$. By definition, the operator norm of $D-$ $\tilde{U}^{\dagger} U^{\prime}$ (with $|\cdot|=\|\cdot\|_{2}$ ) is

$$
\begin{aligned}
\| D & -\tilde{U}^{\dagger} U^{\prime} \|_{\mathrm{op}}^{2} \\
= & \max _{\left|v_{1}\right|^{2}+\left|v_{2}\right|^{2}=1}\left|\left(\begin{array}{cc}
D_{11}-U_{11} & -U_{12} \\
U_{21} & D_{22}-U_{22}
\end{array}\right)\left(\begin{array}{l}
\mathbf{v}_{1} \\
\mathbf{v}_{2}
\end{array}\right)\right|^{2} \\
\leqslant & \left(\left|\left(D_{11}-U_{11}\right) \mathbf{v}_{1}\right|+\left|U_{12} \mathbf{v}_{2}\right|\right)^{2} \\
& +\left(\left|U_{21} \mathbf{v}_{1}\right|+\left|\left(D_{22}-U_{22}\right) \mathbf{v}_{2}\right|\right)^{2} \\
\leqslant & \left(\left\|D_{11}-U_{11}\right\|_{\mathrm{op}}\left|\mathbf{v}_{1}\right|+\left\|U_{12}\right\|_{\mathrm{op}} \sqrt{1-\left|\mathbf{v}_{1}\right|^{2}}\right)^{2} \\
& +\left(\left\|U_{21}\right\|_{\mathrm{op}}\left|\mathbf{v}_{1}\right|+\left\|D_{22}-U_{22}\right\|_{\mathrm{op}} \sqrt{1-\left|\mathbf{v}_{1}\right|^{2}}\right)^{2} \\
= & \left(\left\|D_{11}-U_{11}\right\|_{\mathrm{op}}^{2}+\left\|U_{21}\right\|_{\mathrm{op}}^{2}\right)\left|\mathbf{v}_{1}\right|^{2} \\
& +2\left(\left\|D_{11}-U_{11}\right\|_{\mathrm{op}}\left\|U_{12}\right\|_{\mathrm{op}}\right. \\
& \left.+\left\|U_{21}\right\|_{\mathrm{op}}\left\|D_{22}-U_{22}\right\|_{\mathrm{op}}\right)\left|\mathbf{v}_{1}\right| \sqrt{1-\left|\mathbf{v}_{1}\right|^{2}} \\
& +\left(\left\|U_{12}\right\|_{\mathrm{op}}^{2}+\left\|D_{22}-U_{22}\right\|_{\mathrm{op}}^{2}\right)\left(1-\left|\mathbf{v}_{1}\right|^{2}\right) .
\end{aligned}
$$

The second term of the last expression is maximal at $\left|\mathbf{v}_{1}\right|=\frac{1}{\sqrt{3}}$, the other ones are maximal at $\left|\mathbf{v}_{1}\right|=0,1$, respectively, which allows us to bound

$$
\begin{aligned}
\| D & -\tilde{U}^{\dagger} U^{\prime} \|_{\mathrm{op}}^{2} \\
\leqslant & \frac{2 \sqrt{2}}{3}\left(\left\|D_{11}-U_{11}\right\|_{\mathrm{op}}\left\|U_{12}\right\|_{\mathrm{op}}+\left\|U_{21}\right\|_{\mathrm{op}} \| D_{22}\right. \\
& \left.-U_{22} \|_{\mathrm{op}}\right)+\max \left(\left\|D_{11}-U_{11}\right\|_{\mathrm{op}}^{2}\right. \\
& \left.+\left\|U_{21}\right\|_{\mathrm{op}}^{2},\left\|U_{12}\right\|_{\mathrm{op}}^{2}+\left\|D_{22}-U_{22}\right\|_{\mathrm{op}}^{2}\right) .
\end{aligned}
$$

Using Eq. (23) and $\left\|D_{11}-U_{11}\right\|_{\text {op }},\left\|D_{22}-U_{22}\right\|_{\text {op }}<2$, this implies

$$
\begin{aligned}
\left\|\mathbb{1}-\tilde{U}^{\dagger} U^{\prime}\right\|_{\mathrm{op}}^{2}< & \frac{4 \sqrt{2} c}{3} e^{-\frac{\ell}{\xi_{1}}}+\frac{c^{2}}{4} e^{-\frac{2 \ell}{\xi_{1}}} \\
& +\max \left(\left\|D_{11}-U_{11}\right\|_{\mathrm{op}}^{2},\left\|D_{22}-U_{22}\right\|_{\mathrm{op}}^{2}\right) .
\end{aligned}
$$

Assume now $\left\|D_{11}-U_{11}\right\|_{\text {op }} \geqslant\left\|D_{22}-U_{22}\right\|_{\text {op }}$ (the treatment of the opposite case is analogous). If we decompose $U_{11}$ into blocks according to the invariant subspaces of $\sigma_{2}^{z}$,

$$
U_{11}=\left(\begin{array}{ll}
U_{11,11} & U_{11,12} \\
U_{11,21} & U_{11,22}
\end{array}\right),
$$

Eq. (23) for a $2 \times 2$ block form of $\tilde{U}^{\dagger} U^{\prime}$ with respect to $\sigma_{2}^{z}$ implies $\left\|U_{11,12}\right\|_{\text {op }},\left\|U_{11,21}\right\|_{\text {op }}<\frac{c}{2} e^{-\frac{\ell}{\xi_{2}}}$. This has to hold, because the operator norm of a matrix block cannot be larger than the one of the overall matrix. We can thus repeat the bounding approach of Eqs. (24) and (25) for $\left\|D_{11}-U_{11}\right\|_{\mathrm{op}}^{2}$ and obtain

$$
\begin{aligned}
& \left\|D-\tilde{U}^{\dagger} U^{\prime}\right\|_{\mathrm{op}}^{2}<\frac{4 \sqrt{2} c}{3}\left(e^{-\frac{\ell}{\xi_{1}}}+e^{-\frac{\ell}{\xi_{2}}}\right)+\frac{c^{2}}{4}\left(e^{-\frac{2 \ell}{\xi_{1}}}+e^{-\frac{2 \ell}{\xi_{2}}}\right) \\
& +\max \left(\left\|D_{11,11}-U_{11,11}\right\|_{\mathrm{op}}^{2},\left\|D_{11,22}-U_{11,22}\right\|_{\mathrm{op}}^{2}\right) .
\end{aligned}
$$

Continuation of the same procedure for the remaining sites $i=3,4, \ldots, N$ yields

$$
\left\|D-\tilde{U}^{\dagger} U^{\prime}\right\|_{\mathrm{op}}^{2}<\frac{4 \sqrt{2} c}{3} \sum_{i=1}^{N} e^{-\frac{\ell}{\xi_{i}}}+\frac{c^{2}}{4} \sum_{i=1}^{N} e^{-\frac{2 \ell}{\xi_{i}}}:=\gamma .
$$

This implies $\|[D]_{: n}-\left[\tilde{U}^{\dagger} U^{\prime}\right]_{: n} \mid<\sqrt{\gamma}$, where $[\cdots]_{: n}$ refers to the $n$th column vector. This requires

$$
1-\sqrt{\gamma}<\left|[D]_{: n}\right|=\left|D_{n n}\right|<1+\sqrt{\gamma} .
$$

We choose $\Phi$ such that the diagonal elements $D_{n n}$ of $\tilde{U}^{\dagger} U^{\prime}=$ $\tilde{U}^{\dagger} U \Phi$ are non-negative. Therefore, we obtain using the triangular inequality

$$
\begin{aligned}
& \left\|\mathbb{1}-\tilde{U}^{\dagger} U^{\prime}\right\|_{\mathrm{op}} \\
& \leqslant\|\mathbb{1}-D\|_{\mathrm{op}}+\left\|D-\tilde{U}^{\dagger} U^{\prime}\right\|_{\mathrm{op}} \\
& <2 \sqrt{\gamma}=2 \sqrt{\frac{4 \sqrt{2} c}{3} \sum_{i=1}^{N} e^{-\frac{\ell}{\xi_{i}}}+\frac{c^{2}}{4} \sum_{i=1}^{N} e^{-\frac{2 \ell}{\xi_{i}}} .}
\end{aligned}
$$

Thus, in the limit $\ell / \xi_{\max } \rightarrow \infty$,

$$
\left\|\mathbb{1}-\tilde{U}^{\dagger} U^{\prime}\right\|_{\mathrm{op}}<4 \sqrt{\frac{\sqrt{2} c}{3} N e^{-\frac{\ell}{2 \xi_{\max }}}} .
$$

For $\ell=\alpha N$ and $\xi_{\max }<c^{\prime} N^{1-\mu}$ we arrive at

$$
\left\|U^{\prime}-\tilde{U}\right\|_{\mathrm{op}}<2^{9 / 4} \sqrt{\frac{c N}{3}} e^{-\frac{\alpha N^{\mu}}{2 c^{\prime}}} .
$$

We $\quad$ set $\quad \delta(N):=2^{9 / 4} \sqrt{\frac{c N}{3}} e^{-\frac{\alpha N^{\mu}}{2 c^{\prime}}} \quad$ with obviously $\lim _{N \rightarrow \infty} \delta(N)=0$. Next, we derive the implications of time reversal symmetry on the unitary matrix $U^{\prime}$. (For simplicity, we drop the prime from now on.) By its definition,

$$
U^{\dagger} H U=E,
$$

where $E$ is the diagonal matrix containing the corresponding energy eigenvalues. Condition 2 says $H=v^{\otimes N} H^{*}\left(v^{\dagger}\right)^{\otimes N}$, i.e., we have

$$
U^{\dagger} \mathcal{V} H^{*} \mathcal{V}^{\dagger} U=E
$$

where in the following we use the symbol $\mathcal{V}$ in a slightly sloppy way denoting the right number of tensor products of unitaries $v\left(\right.$ i.e., here $\mathcal{V}=v^{\otimes N}$ ). Due to $H^{*}=U^{*} E U^{\top}$ we obtain

$$
U^{\dagger} \mathcal{V} U^{*} E U^{\top} \mathcal{V}^{\dagger} U=E \text {. }
$$

For spin systems, time reversal symmetry does not protect any degeneracies. Condition 3 allows us to add a local perturbation that breaks any remaining accidental degeneracies for finite $N$, such as $V=\sum_{i} h_{i}^{\prime} \sigma_{z}^{i}$ with random $h_{i}^{\prime}$ for Hamiltonian Eq. (1). Hence, we can assume that there are no degeneracies, such that Eq. (36) implies

$$
\Theta=U^{\dagger} \mathcal{V} U^{*},
$$

where $\Theta$ is a diagonal matrix with diagonal elements of magnitude 1 . Using Lemma 1 , the triangular inequality yields

$$
\begin{aligned}
\left\|\tilde{U} \Theta-\mathcal{V} \tilde{U}^{*}\right\|_{\text {op }} \leqslant & \left\|U \Theta-\mathcal{V} U^{*}\right\|_{\text {op }}+\|(\tilde{U}-U) \Theta\|_{\text {op }} \\
& +\left\|\mathcal{V}\left(U^{*}-\tilde{U}^{*}\right)\right\|_{\mathrm{op}} \\
< & 0+\delta(N)\|\Theta\|_{\mathrm{op}}+\delta(N)\|\mathcal{V}\|_{\mathrm{op}} \\
= & 2 \delta(N) .
\end{aligned}
$$

We now proceed to prove the following:

Lemma 2. $\left\|\tilde{U} \Theta-\mathcal{V} \tilde{U}^{*}\right\|_{\text {op }}<2 \delta(N)$ implies that there exists a two-layer quantum circuit $\bar{U}$ with gates of length $4 \ell$ such that to leading order $\left\|\bar{U}-\mathcal{V} \bar{U}^{*}\right\|_{\text {op }}<(2+4 N) \delta(N)$. 
Proof of Lemma 2.First, we note that Eq. (38) implies in graphical notation
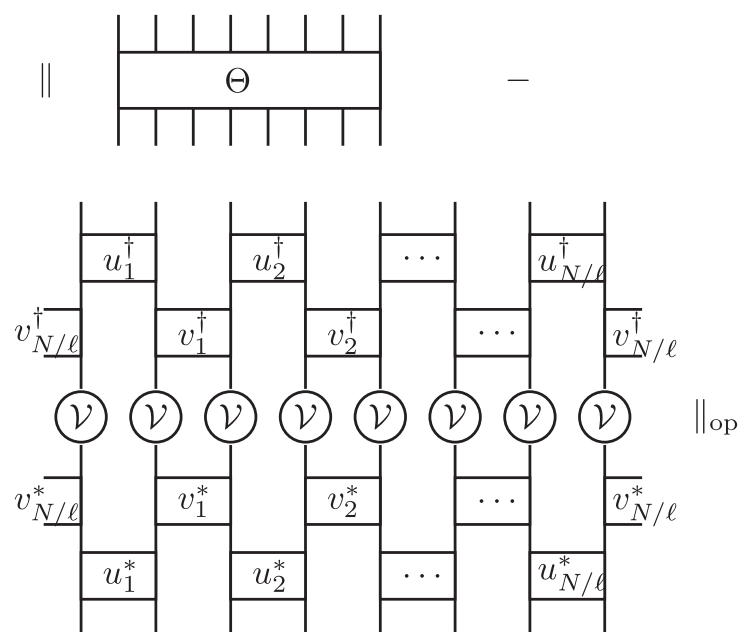

$<2 \delta(N)$ where each leg corresponds to dimension $2^{\ell / 2}$. Let us combine $v_{k}^{\dagger} \mathcal{V} v_{k}^{*}:=V_{k}$ and define $\mathbf{l}_{k}:=\left(l_{\ell(k-1)+1}, l_{\ell(k-1)+2}, \ldots, l_{\ell k}\right)$. The diagonal elements of the left side of Eq. (39) are bound by $\left|d_{\mathbf{l}_{1} \cdots \mathbf{l}_{\frac{N}{\ell}}}\right|<2 \delta(N)$ with

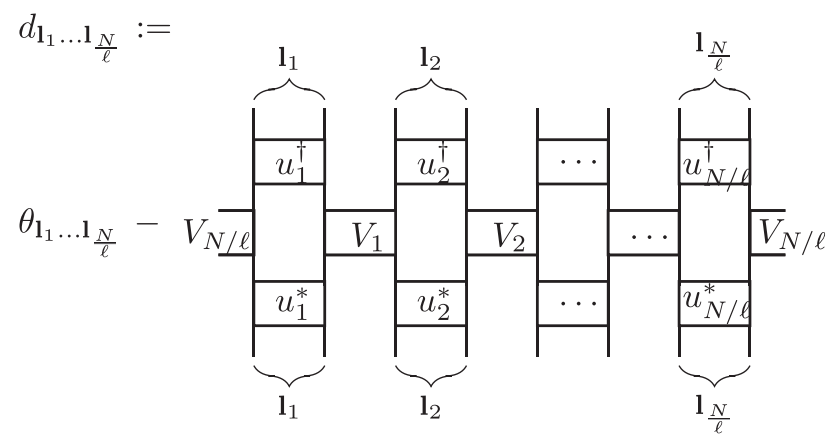

(40)

where $\theta_{\mathbf{l}_{1} \cdots \mathbf{l}_{\frac{N}{\ell}}}$ are the diagonal elements of $\Theta$. Curly braces denote the corresponding choice of 1-bits. We define $F_{k}:=$ $\left|\mathbf{l}_{k}\right\rangle\left\langle\mathbf{l}_{k}^{\prime}\right|$ and obtain further

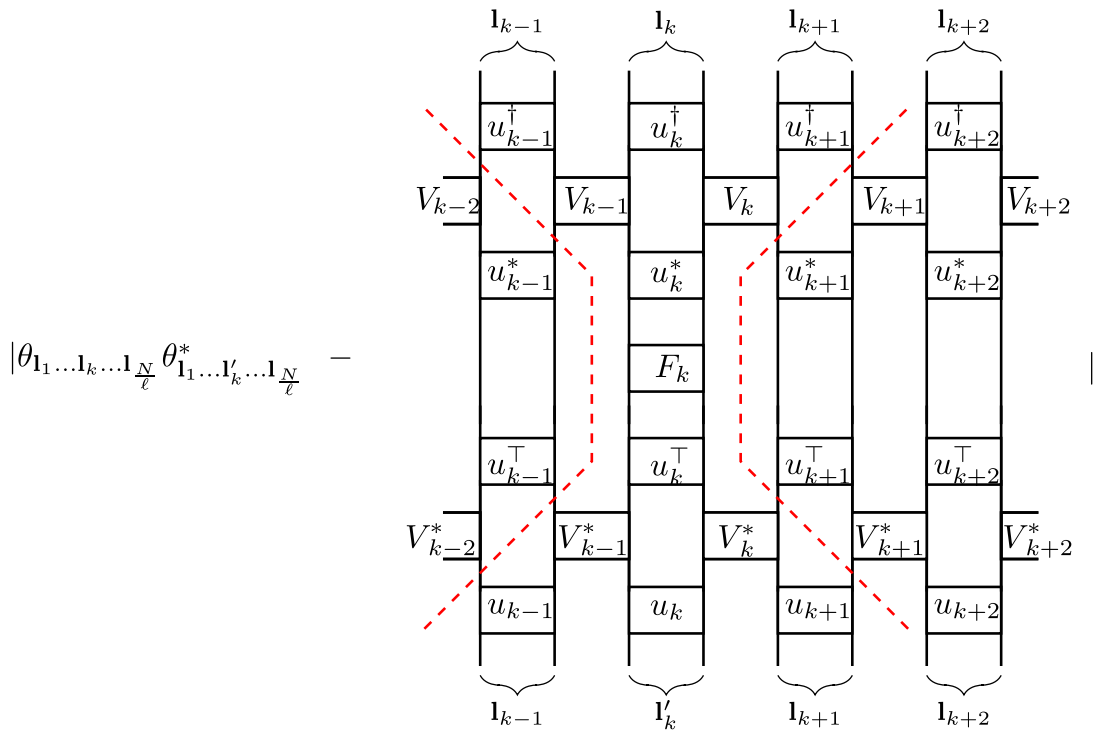

$$
\begin{aligned}
& =\left|\theta_{\mathbf{l}_{1} \ldots \mathbf{1}_{k} \ldots \mathbf{1}_{\frac{N}{\ell}}} \theta_{\mathbf{l}_{1} \ldots \mathbf{1}_{k}^{\prime} \ldots \mathbf{l}_{\frac{N}{\ell}}^{*}}-\left(\theta_{\mathbf{l}_{1} \ldots \mathbf{1}_{k} \ldots \mathbf{1}_{\frac{N}{\ell}}}-d_{\mathbf{l}_{1} \ldots \mathbf{l}_{k} \ldots \mathbf{1}_{\frac{N}{\ell}}}\right)\left(\theta_{\mathbf{l}_{1} \ldots \mathbf{1}_{k}^{\prime} \ldots \mathbf{1}_{\frac{N}{\ell}}^{*}}-d_{\mathbf{l}_{1} \ldots \mathbf{l}_{k} \ldots \mathbf{1}_{\frac{N}{\ell}}^{\prime}}^{*}\right)\right| \\
& =\left|\theta_{\mathbf{l}_{1} \ldots \mathbf{1}_{k} \ldots \mathbf{1}_{\frac{N}{\ell}}} d_{\mathbf{l}_{1} \ldots \mathbf{1}_{k}^{\prime} \ldots \mathbf{1}_{\frac{N}{\ell}}^{*}}+\theta_{\mathbf{l}_{1} \ldots \mathbf{1}_{k}^{\prime} \ldots \mathbf{1}_{\frac{N}{\ell}}}^{*} d_{\mathbf{l}_{1} \ldots \mathbf{l}_{k} \ldots \mathbf{1}_{\frac{N}{\ell}}}-d_{\mathbf{l}_{1} \ldots \mathbf{1}_{k} \ldots \mathbf{1}_{\frac{N}{\ell}}} d_{\mathbf{l}_{1} \ldots \mathbf{1}_{k}^{\prime} \ldots \mathbf{1}_{\frac{N}{\ell}}}\right| \\
& <4 \delta(N)+[2 \delta(N)]^{2} \rightarrow 4 \delta(N)
\end{aligned}
$$

to leading order. All unitaries outside the causal cone marked by red dashed lines cancel in Eq. (41), yielding identities. Thus,

$$
\left|\theta_{\mathbf{l}_{1} \cdots \mathbf{l}_{k} \cdots \mathbf{l}_{\frac{N}{\ell}}} \theta_{\mathbf{l}_{1} \cdots \mathbf{l}_{k}^{\prime} \cdots \mathbf{l}_{\frac{N}{\ell}}}^{*}-e^{i g_{k}\left(\mathbf{l}_{k-1}, \mathbf{l}_{k}, \mathbf{l}_{k}^{\prime}, \mathbf{l}_{k+1}\right)}\right|<4 \delta(N)
$$

with discrete functions (tensors) $g_{k} \in \mathbb{C}$ depending only on the local l-bit configuration. (We define $k$ modulo $\frac{N}{\ell}$, i.e., $\frac{N}{\ell}+1 \equiv 1$.) Note that Eq. (42) allows for a small imaginary part in $g_{k}$. If we now set $\theta_{\mathbf{l}_{1} \cdots \mathbf{l}_{k} \cdots \mathbf{l}_{\frac{N}{\ell}}}:=e^{i f\left(\mathbf{l}_{1}, \ldots, \mathbf{l}_{k}, \ldots, \mathbf{l}_{\frac{N}{\ell}}\right)}$ with $f_{k} \in \mathbb{R}$ and define

$$
f\left(\mathbf{l}_{1}, \ldots, \mathbf{l}_{k}, \ldots, \mathbf{l}_{\frac{N}{\ell}}\right)-f\left(\mathbf{l}_{1}, \ldots, \mathbf{l}_{k}^{\prime}, \ldots, \mathbf{l}_{\frac{N}{\ell}}\right)-g_{k}\left(\mathbf{l}_{k-1}, \mathbf{l}_{k}, \mathbf{l}_{k}^{\prime}, \mathbf{l}_{k+1}\right) \bmod 2 \pi:=\epsilon_{k}\left(\left\{\mathbf{l}, \mathbf{l}^{\prime}\right\}\right),
$$


we obtain to lowest order $\left|\epsilon_{k}\left(\left\{\mathbf{I}, \mathbf{I}^{\prime}\right\}\right)\right|<4 \delta(N)$. We reuse this equation now by consecutively switching l-bits, $\mathbf{l}_{k+1}$ to $\mathbf{l}_{k+1}^{\prime}, \mathbf{l}_{k+2}$ to $\mathbf{l}_{k+2}^{\prime}$, etc. until we reach $\mathbf{l}_{k-1}$ (through the periodic boundary). That is,

$$
\begin{array}{r}
f\left(\mathbf{l}_{1}, \ldots, \mathbf{l}_{k-1}, \mathbf{l}_{k}, \mathbf{l}_{k+1}, \mathbf{l}_{k+2} \ldots, \mathbf{l}_{\frac{N}{\ell}}\right)-f\left(\mathbf{l}_{1}, \ldots, \mathbf{l}_{k-1}, \mathbf{l}_{k}^{\prime}, \mathbf{l}_{k+1}, \mathbf{l}_{k+2}, \ldots, \mathbf{l}_{\frac{N}{\ell}}\right)-g_{k}\left(\mathbf{l}_{k-1}, \mathbf{l}_{k}, \mathbf{l}_{k}^{\prime}, \mathbf{l}_{k+1}\right)=\epsilon_{k}\left(\left\{\mathbf{l}, \mathbf{l}^{\prime}\right\}\right) \\
f\left(\mathbf{l}_{1}, \ldots, \mathbf{l}_{k-1}, \mathbf{l}_{k}^{\prime}, \mathbf{l}_{k+1}, \mathbf{l}_{k+2} \ldots, \mathbf{l}_{\frac{N}{\ell}}\right)-f\left(\mathbf{l}_{1}, \ldots, \mathbf{l}_{k-1}, \mathbf{l}_{k}^{\prime}, \mathbf{l}_{k+1}^{\prime}, \mathbf{l}_{k+2}, \ldots, \mathbf{l}_{\frac{N}{\ell}}\right)-g_{k+1}\left(\mathbf{l}_{k}^{\prime}, \mathbf{l}_{k+1}, \mathbf{l}_{k+1}^{\prime}, \mathbf{l}_{k+2}\right)=\epsilon_{k+1}\left(\left\{\mathbf{l}, \mathbf{l}^{\prime}\right\}\right) \\
f\left(\mathbf{l}_{1}, \ldots, \mathbf{l}_{k-1}, \mathbf{l}_{k}^{\prime}, \mathbf{l}_{k+1}^{\prime}, \mathbf{l}_{k+2} \ldots, \mathbf{l}_{\frac{N}{\ell}}\right)-f\left(\mathbf{l}_{1}, \ldots, \mathbf{l}_{k-1}, \mathbf{l}_{k}^{\prime}, \mathbf{l}_{k+1}^{\prime}, \mathbf{l}_{k+2}^{\prime}, \ldots, \mathbf{l}_{\frac{N}{\ell}}\right)-g_{k+2}\left(\mathbf{l}_{k+1}^{\prime}, \mathbf{l}_{k+2}, \mathbf{l}_{k+2}^{\prime}, \mathbf{l}_{k+3}\right)=\epsilon_{k+2}\left(\left\{\mathbf{l}, \mathbf{l}^{\prime}\right\}\right) \\
\ldots \\
f\left(\mathbf{l}_{1}^{\prime}, \ldots, \mathbf{l}_{k-1}, \mathbf{l}_{k}^{\prime}, \mathbf{l}_{k+1}^{\prime}, \mathbf{l}_{k+2}^{\prime} \ldots, \mathbf{l}_{\frac{N}{\ell}}^{\prime}\right)-f\left(\mathbf{l}_{1}^{\prime}, \ldots, \mathbf{l}_{k-1}^{\prime}, \mathbf{l}_{k}^{\prime}, \mathbf{l}_{k+1}^{\prime}, \mathbf{l}_{k+2}^{\prime}, \ldots, \mathbf{l}_{\frac{N}{\ell}}^{\prime}\right)-g_{k-1}\left(\mathbf{l}_{k-2}^{\prime}, \mathbf{l}_{k-1}, \mathbf{l}_{k-1}^{\prime}, \mathbf{l}_{k}^{\prime}\right)=\epsilon_{k-1}\left(\left\{\mathbf{l}, \mathbf{l}^{\prime}\right\}\right) .
\end{array}
$$

If we add all left and right sides together, we arrive at

$$
\begin{aligned}
& \mid f\left(\mathbf{l}_{1}, \ldots, \mathbf{l}_{k}, \ldots, \mathbf{l}_{\frac{N}{\ell}}\right)-f\left(\mathbf{l}_{1}^{\prime}, \ldots, \mathbf{l}_{k}^{\prime}, \ldots, \mathbf{l}_{\frac{N}{\ell}}^{\prime}\right)-g_{k}\left(\mathbf{l}_{k-1}, \mathbf{l}_{k}, \mathbf{l}_{k}^{\prime}, \mathbf{l}_{k+1}\right) \\
& \quad-\sum_{m \in\left\{k+1, k+2, \ldots, \frac{N}{\ell}, 1,2, \ldots, k-2\right\}} g_{m}\left(\mathbf{l}_{m-1}^{\prime}, \mathbf{l}_{m}, \mathbf{l}_{m}^{\prime}, \mathbf{l}_{m+1}\right)-g_{k-1}\left(\mathbf{l}_{k-2}^{\prime}, \mathbf{l}_{k-1}, \mathbf{l}_{k-1}^{\prime}, \mathbf{l}_{k}^{\prime}\right) \mid<4 N \delta(N) .
\end{aligned}
$$

We fix now $\mathbf{l}_{1}^{\prime}=\mathbf{l}_{2}^{\prime}=\cdots=\mathbf{l}_{N / \ell}^{\prime}=\mathbf{0}$ such that

$$
\begin{aligned}
& \mid f\left(\mathbf{l}_{1}, \ldots, \mathbf{l}_{N / \ell}\right)-f(\mathbf{0}, \ldots, \mathbf{0})-g_{k}\left(\mathbf{l}_{k-1}, \mathbf{l}_{k}, \mathbf{0}, \mathbf{l}_{k+1}\right) \\
& \quad-\sum_{m \in\left\{k+1, k+2, \ldots, \frac{N}{\ell}, 1,2, \ldots, k-2\right\}} g_{m}\left(\mathbf{0}, \mathbf{l}_{m}, \mathbf{0}, \mathbf{l}_{m+1}\right)-g_{k-1}\left(\mathbf{0}, \mathbf{l}_{k-1}, \mathbf{0}, \mathbf{0}\right) \mid<4 N \delta(N) .
\end{aligned}
$$

Since the choice of $k$ is arbitrary, this implies there are discrete functions $\bar{g}_{m}\left(\mathbf{l}_{m}, \mathbf{l}_{m+1}\right) \in \mathbb{C}$ such that

$$
\left|f\left(\mathbf{l}_{1}, \ldots, \mathbf{l}_{N / \ell}\right)-\sum_{m=1}^{N / \ell} \bar{g}_{m}\left(\mathbf{l}_{m}, \mathbf{l}_{m+1}\right)\right|<4 N \delta(N),
$$

which also means

$$
\left|f\left(\mathbf{l}_{1}, \ldots, \mathbf{l}_{N / \ell}\right)-\sum_{m=1}^{N / \ell} \operatorname{Re}\left[\bar{g}_{m}\left(\mathbf{l}_{m}, \mathbf{l}_{m+1}\right)\right]\right|<4 N \delta(N),
$$

We now define $\bar{\Theta}_{m}$ as the diagonal matrix acting on 1-bits $\mathbf{I}_{m}$, $\mathbf{l}_{m+1}$ with diagonal elements $e^{\frac{i}{2} \operatorname{Re}\left[\bar{g}_{m}\left(\mathbf{l}_{m}, \mathbf{l}_{m+1}\right)\right]}$. We can therefore write
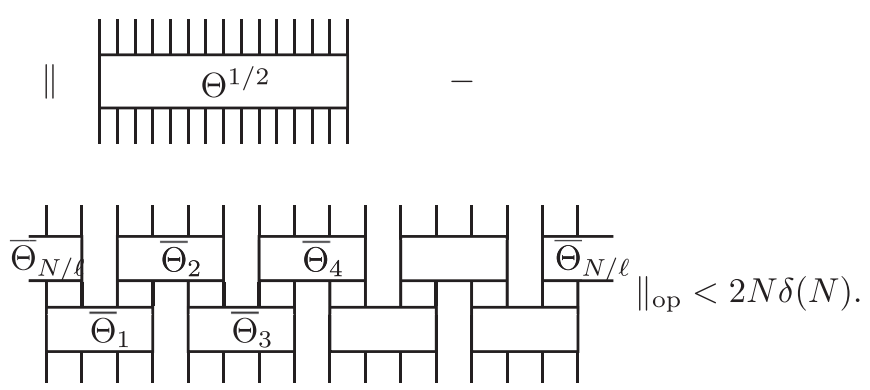

Let us define

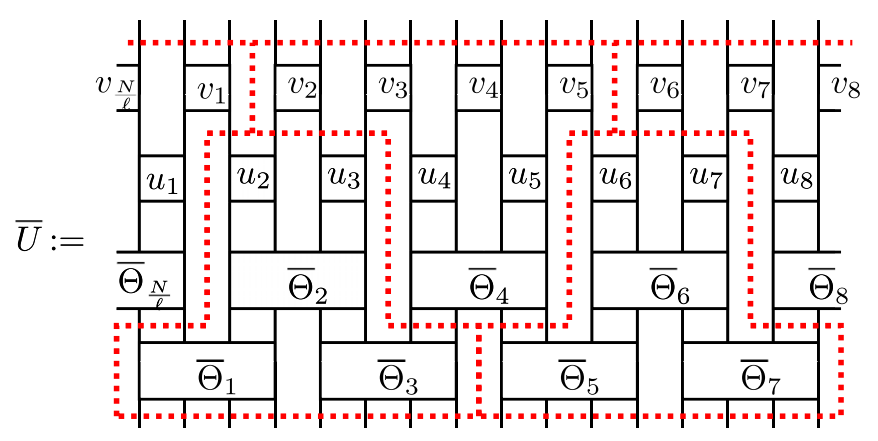

(49)

We combine unitaries as indicated by the red dotted lines to new unitaries $\bar{u}_{k}, \bar{v}_{k}$ acting on $4 \ell$ sites,

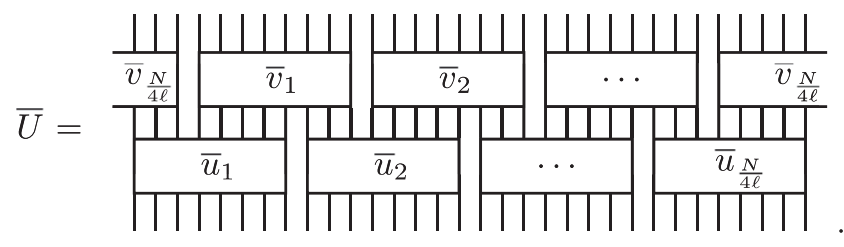

(50)

Equation (48) implies $\left\|\tilde{U} \Theta^{\frac{1}{2}}-\bar{U}\right\|_{\text {op }}<2 N \delta(N)$. The new quantum circuit has the same structure as the original one apart from the fact that the upper layer of unitaries is displaced by $\ell / 2$ sites. Finally, we obtain using the triangular inequality

$$
\begin{aligned}
\left\|\bar{U}-\mathcal{V} \bar{U}^{*}\right\|_{\mathrm{op}} \leqslant & \left\|\tilde{U} \Theta^{\frac{1}{2}}-\mathcal{V} \tilde{U}^{*} \Theta^{-\frac{1}{2}}\right\|_{\mathrm{op}}+\left\|\bar{U}-\tilde{U} \Theta^{\frac{1}{2}}\right\|_{\mathrm{op}} \\
& +\left\|\mathcal{V}\left(\tilde{U}^{*} \Theta^{-\frac{1}{2}}-\bar{U}^{*}\right)\right\|_{\mathrm{op}}<2 \delta(N) \\
& +2 N \delta(N)+2 N \delta(N) .
\end{aligned}
$$


From the preceding discussion we gather that $\Theta$ cannot be absorbed into the definition of $\tilde{U}$ in the case of an onsite symmetry (instead of time reversal symmetry), which prevents extension of the current proof to all one-dimensional symmetry-protected MBL phases.

Let us now define $\delta_{1}(N)=(2+4 N) \delta(N)$ (which also vanishes in the limit $N \rightarrow \infty$ ). In the new quantum circuit, each unitary $\bar{u}_{k}$ has $3 \ell / 2$ (upper and lower) left legs and $5 \ell / 2$ right legs and vice versa for the $\bar{v}_{k}$ 's.

We now prove the following:

Lemma 3. If $\left\|\bar{U}-\mathcal{V} \bar{U}^{*}\right\|_{\mathrm{op}}<\delta_{1}(N)$, the unitaries $\bar{u}_{k}$ and $\bar{v}_{k}$ fulfill

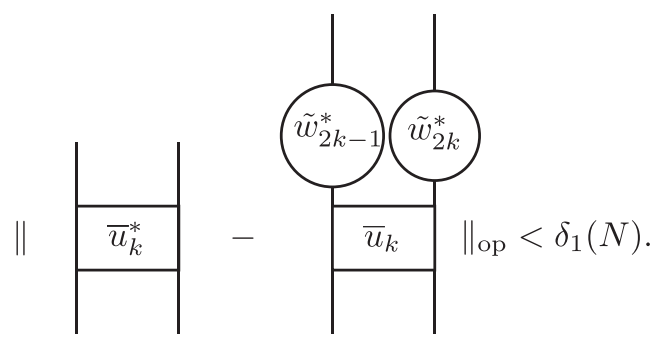

and

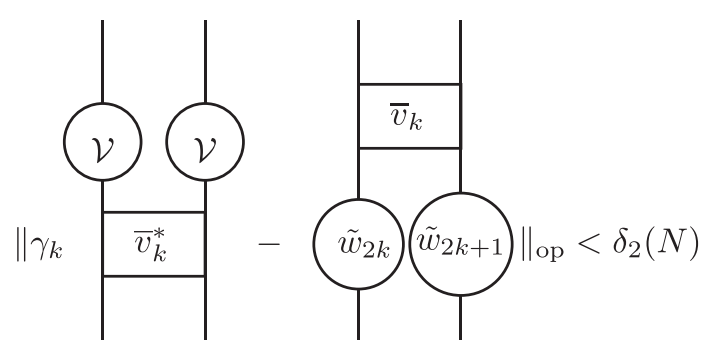

with $\delta_{2}(N)=3 \delta_{1}(N)+4 n \delta_{1}(N), \gamma_{k} \in \mathbb{C}$ with $\left|\gamma_{k}\right| \rightarrow 1$ to zeroth order in $N$ and matrices $\tilde{w}_{j}$ having the property

$$
\left\|\mathbb{1} \mp \tilde{w}_{j} \tilde{w}_{j}^{*}\right\|_{\mathrm{op}}<11 \delta_{1}(N)
$$

Proof of Lemma 3. After setting $\bar{u}_{k}^{\prime}=\bar{u}_{k}^{*}$ and $\bar{v}_{k}^{\prime}=\mathcal{V} \bar{v}_{k}^{*}$, Lemma 2 implies

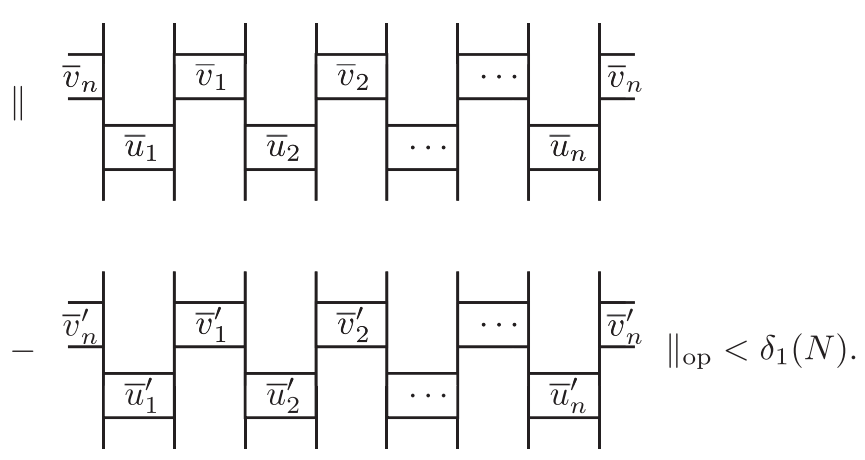

Since the operator norm is multiplicative (and 1 for unitary matrices), we can insert a tensor product of all $\bar{u}_{k}^{\prime \dagger}$ from the bottom and one of all $\bar{v}_{k}^{\dagger}$ from the top inside the operator norm to obtain

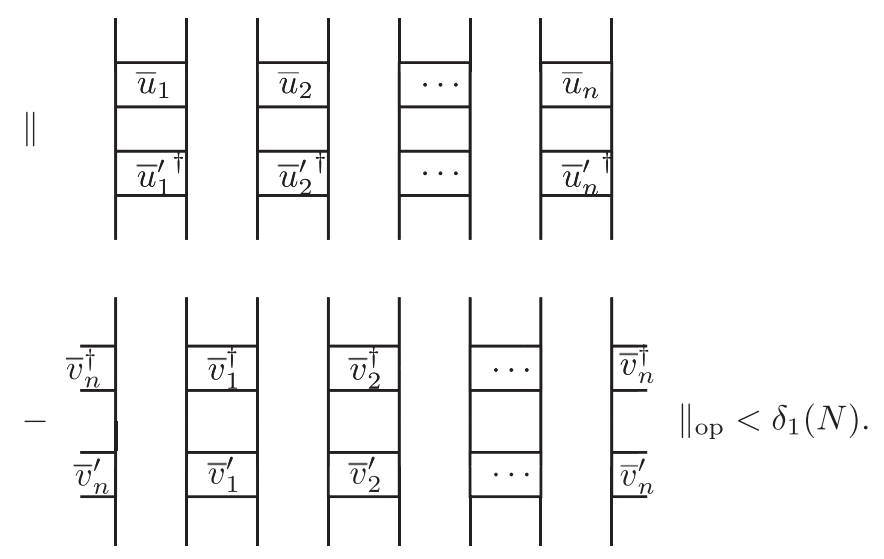

Therefore, since $n=\frac{N}{4 \ell}=\frac{1}{4 \alpha}$ is fixed, in the limit $N \rightarrow \infty$, both sides must become a tensor product of unitaries $w_{j}$,

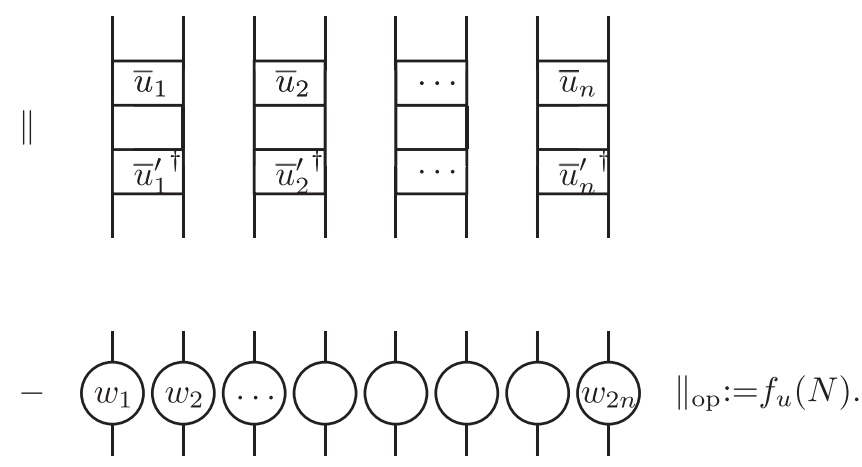

and

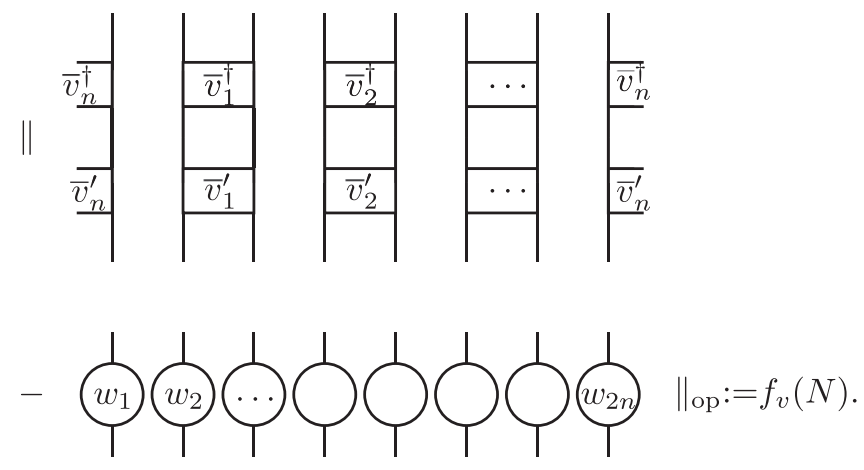

with $\lim _{N \rightarrow \infty} f_{u}(N)=\lim _{N \rightarrow \infty} f_{v}(N)=0$. We now multiply Eq. (56) from the top by $\otimes_{j=1}^{2 n} w_{j}^{\dagger}$ and fix some of the upper and lower indices to be $\bar{l}_{j}$ (which is defined to correspond to a group of indices, $\quad \bar{l}_{2 k-1}=\left(l_{4 \ell(k-1)+1}, l_{4 \ell(k-1)+2}, \ldots, l_{4 \ell(k-1)+\frac{3}{2} \ell}\right)$, $\bar{l}_{2 k}=\left(l_{4 \ell(k-1)+\frac{3}{2} \ell+1}, l_{4 \ell(k-1)+\frac{3}{2} \ell+2}, \ldots, l_{4 \ell k}\right) \quad$ with 
$k=1,2, \ldots, n)$,

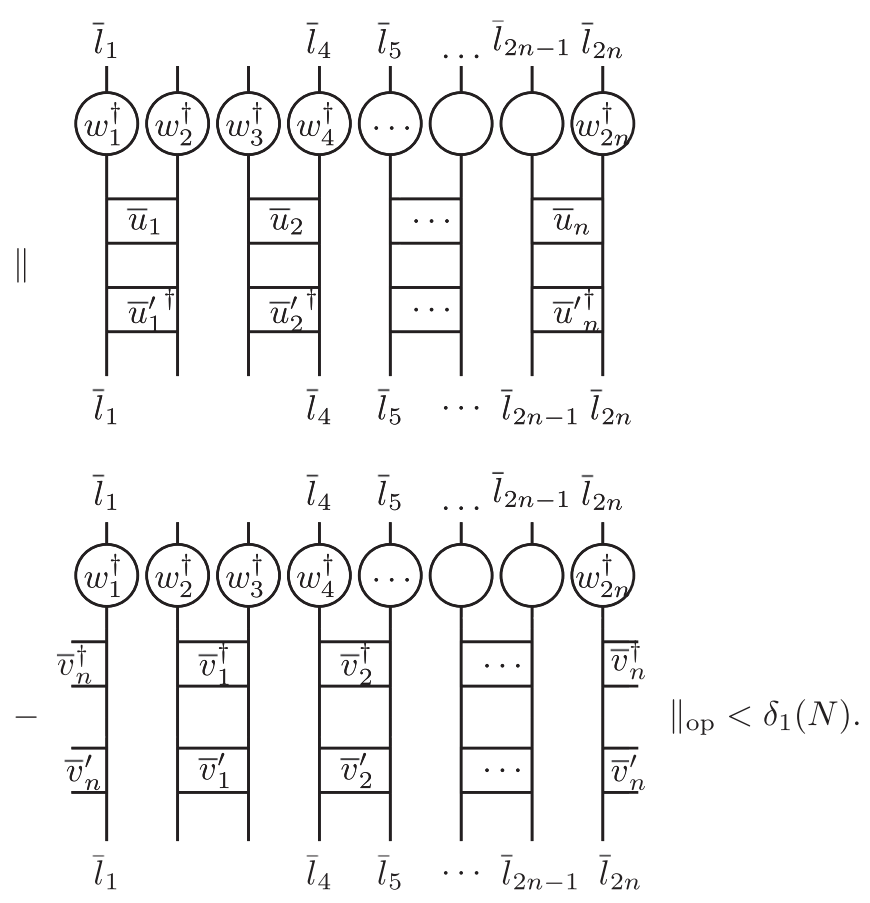

(59)

This is true because the operator norm is also bounded for subblocks. In order to bound the scalar factors above, let us fix all indices of Eqs. (57) and (58) (after also multiplying by $\otimes_{j=1}^{2 n} w_{j}^{\dagger}$ from the top),

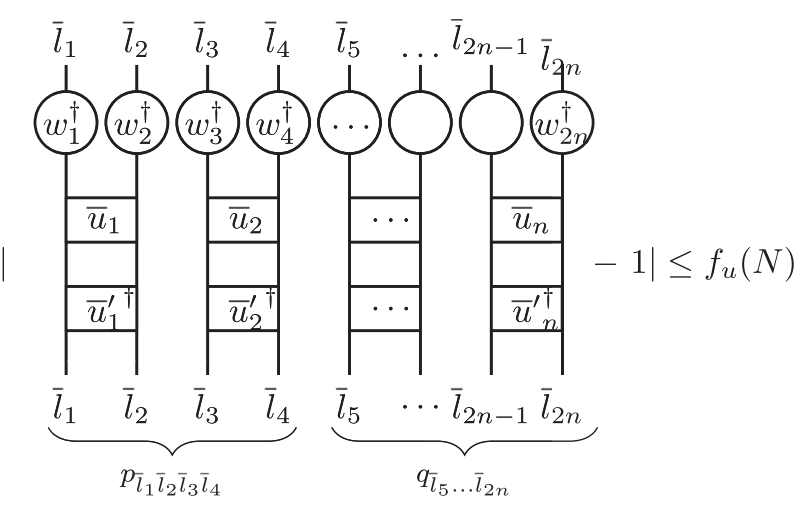

and

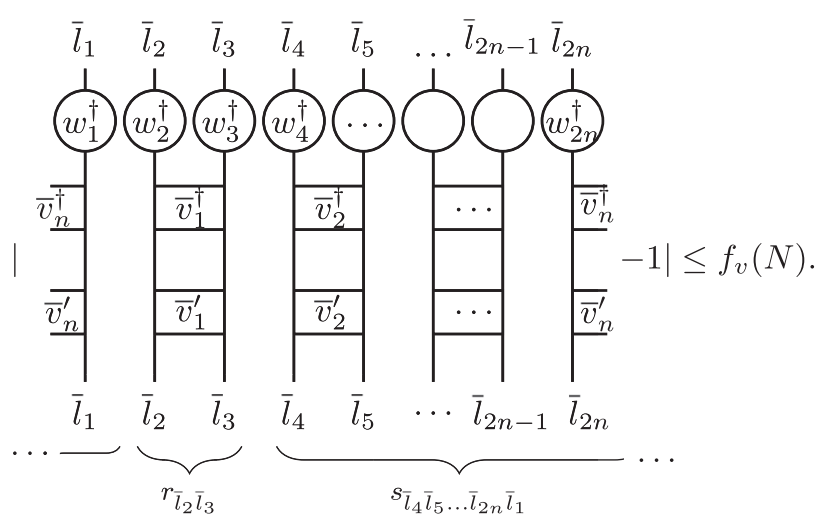

The absolute values of the factors indicated by curly braces are all upper bounded by 1 , which implies

$$
\begin{gathered}
1-\left|q_{\bar{l}_{5} \ldots \bar{l}_{2 n}}\right| \leqslant f_{u}(N), \\
1-\left|s_{\bar{l}_{4} \ldots \bar{l}_{2 n} \bar{l}_{1}}\right| \leqslant f_{v}(N) .
\end{gathered}
$$

First, by definition of $q_{\bar{l}_{5} \ldots \bar{l}_{2 n}}$ and $s_{\bar{l}_{4} \ldots \bar{l}_{2 n} \bar{l}_{1}}$, Eq. (59) is equivalent to

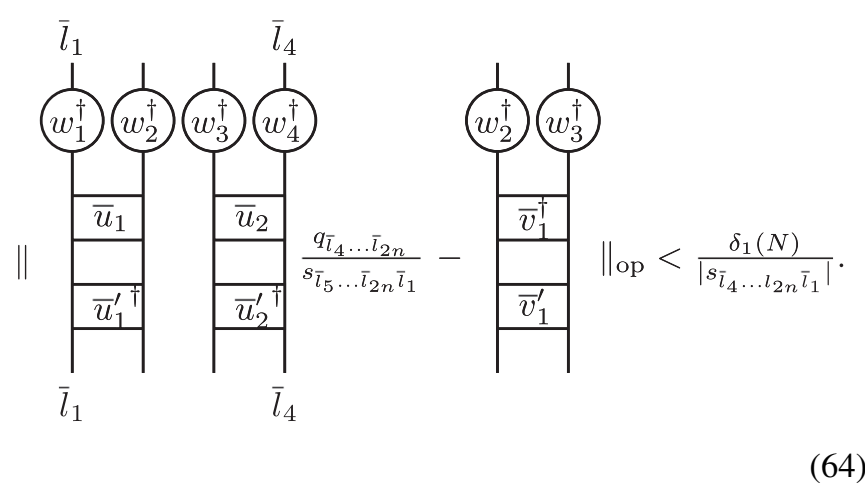

The left term is a tensor product of two matrices $x_{2}, x_{3}$ which are not necessarily unitary and fulfill

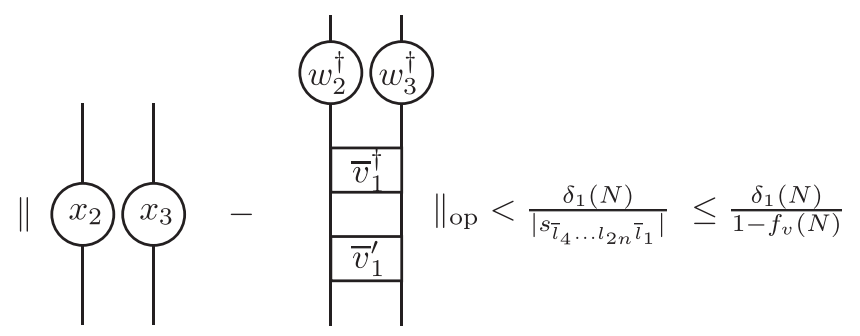

using Eq. (61). Without loss of generality we can set $\left\|x_{2}\right\|_{\text {op }}=$ $\left\|x_{3}\right\|_{\mathrm{op}}$. Following the same line of reasoning for the other $v$ unitaries (and also $u$ unitaries), we arrive at

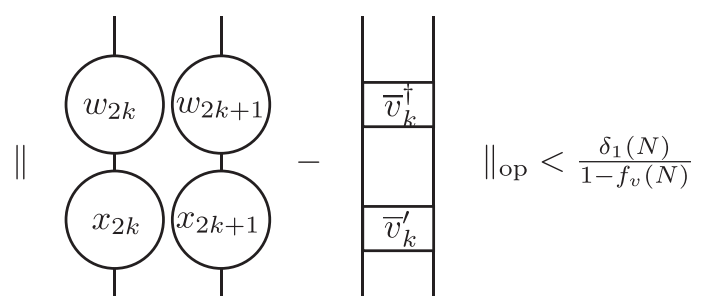

and

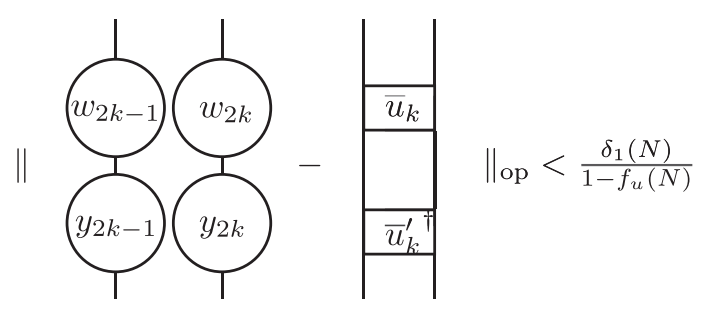

with $\left\|x_{2 k}\right\|_{\mathrm{op}}=\left\|x_{2 k+1}\right\|_{\mathrm{op}}$ and $\left\|y_{2 k-1}\right\|_{\mathrm{op}}=\left\|y_{2 k}\right\|_{\mathrm{op}}$, where the $y_{j}$ again need not be unitaries. The left sides of Eqs. (66) and (67) are thus to leading order bound by $\delta_{1}(N)$. Hence, 
if we define difference tensors $R_{k}, S_{k}$ via

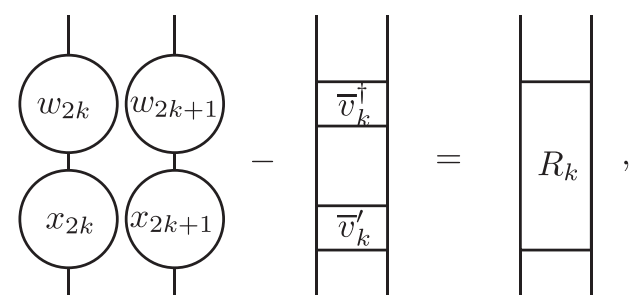

and

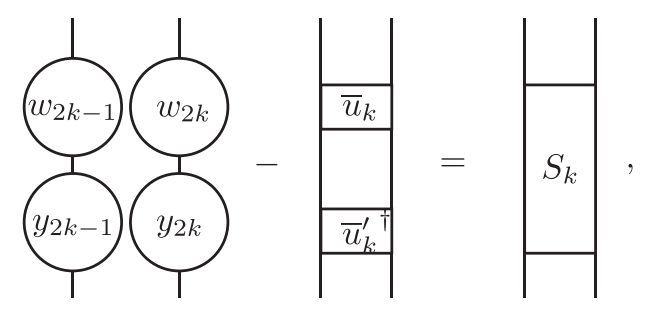

their operator norms are (to leading order) bounded by $\left\|R_{k}\right\|_{\text {op }},\left\|S_{k}\right\|_{\text {op }}<\delta_{1}(N)$. Using Eqs. (68) and (69), we obtain using the triangular inequality
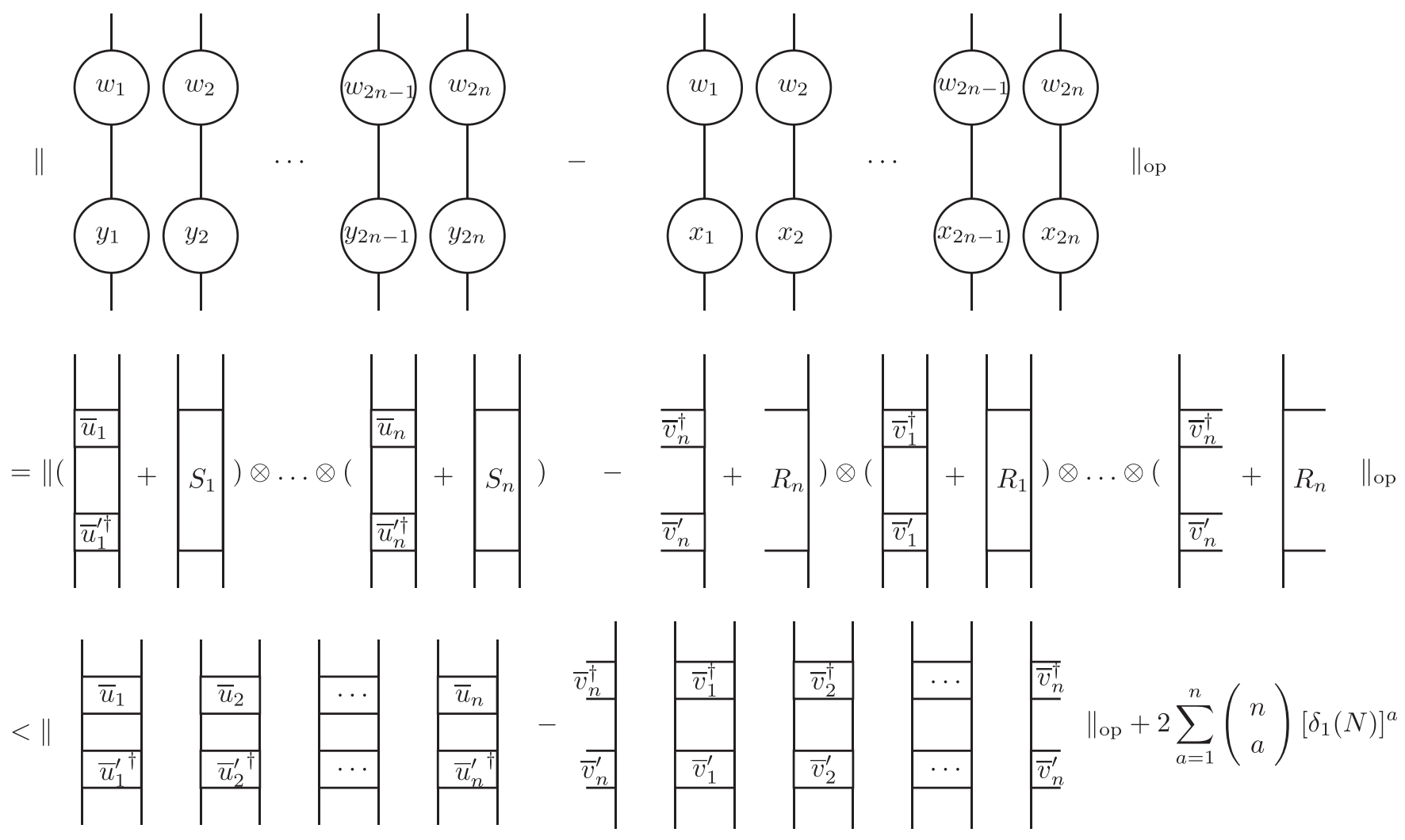

$\rightarrow \delta_{1}(N)+2 n \delta_{1}(N)$

(70)

to leading order. Hence, there have to be scalars $\sigma_{j} \in \mathbb{C}$ such that

$$
\left\|x_{j} \sigma_{j}-y_{j}\right\|_{\text {op }}<\delta_{1}(N)+2 n \delta_{1}(N) .
$$

Because of Eqs. (66) and (67), $x_{j}$ and $y_{j}$ are unitaries to zeroth order in $N$. We now define $w_{j} y_{j}:=\tilde{w}_{j}$ (i.e., $\left\|\tilde{w}_{2 k-1}\right\|_{\text {op }}=$ $\left\|\tilde{w}_{2 k}\right\|_{\text {op }}$ ), such that Eq. (67) reads (to leading order)

$$
\|_{\text {op }}<\delta_{1}(N) .
$$

From this inequality, we obtain

$$
\begin{aligned}
\left\|\tilde{w}_{2 k-1}\right\|_{\mathrm{op}}^{2} & =\left\|\tilde{w}_{2 k}\right\|_{\mathrm{op}}^{2}=\left\|\tilde{w}_{2 k-1} \otimes \tilde{w}_{2 k}\right\|_{\mathrm{op}} \\
& \leqslant\left\|\tilde{w}_{2 k-1} \otimes \tilde{w}_{2 k}-\bar{u}_{k} \bar{u}_{k}^{\prime \dagger}\right\|_{\mathrm{op}}+\left\|\bar{u}_{k} \bar{u}_{k}^{\prime \dagger}\right\|_{\mathrm{op}} \\
& <\delta_{1}(N)+1
\end{aligned}
$$

and

$$
\begin{aligned}
1 & =\left\|\bar{u}_{k} \bar{u}_{k}^{\prime \dagger}\right\|_{\mathrm{op}} \\
& \leqslant\left\|\bar{u}_{k} \bar{u}_{k}^{\dagger}-\tilde{w}_{2 k-1} \otimes \tilde{w}_{2 k}\right\|_{\mathrm{op}}+\left\|\tilde{w}_{2 k-1} \otimes \tilde{w}_{2 k}\right\|_{\mathrm{op}} \\
& <\delta_{1}(N)+\left\|\tilde{w}_{2 k-1}\right\|_{\mathrm{op}}^{2} .
\end{aligned}
$$

Equations (73) and (74) together yield to leading order

$$
\left|1-\left\|\tilde{w}_{j}\right\|_{\text {op }}\right|<\frac{1}{2} \delta_{1}(N) .
$$


In order to derive a similar relation for the $\bar{v}_{k}$ matrices, let us consider the following property: Assume $\|A-B C\|<\epsilon_{1}$, $\|B-D\|<\epsilon_{2}$ and $\|C-E\|<\epsilon_{3}$ (in any norm). If we now set $\Delta:=B-D$ and $\Delta^{\prime}:=C-E$, we find

$$
\begin{aligned}
\|A-D E\|= & \left\|A-(B-\Delta)\left(C-\Delta^{\prime}\right)\right\| \\
< & \|A-B C\|+\|\Delta\|\|C\|+\left\|\Delta^{\prime}\right\|\|B\| \\
& +\|\Delta\|\left\|\Delta^{\prime}\right\|<\epsilon_{1}+\epsilon_{2}\|C\|+\epsilon_{3}\|B\|+\epsilon_{2} \epsilon_{3},
\end{aligned}
$$

where the last term is of subleading order. Because of $\left\|w_{j} x_{j} \sigma_{j}-\tilde{w}_{j}\right\|_{\text {op }}<\delta_{1}(N)+2 n \delta_{1}(N)$ [from Eq. (71)] we thus have

$$
\begin{aligned}
& \underbrace{}_{-1} \sigma_{\tilde{w}_{2 k}} \sigma_{2 k} \sigma_{2 k+1} \|_{\mathrm{op}} \\
& \|_{\text {op } \sigma_{2 k} \sigma_{2 k+1}} \\
& +\left(\delta_{1}(N)+2 n \delta_{1}(N)\right)\left(\left\|\tilde{w}_{2 k}\right\|_{\text {op }}+\left\|\tilde{w}_{2 k+1}\right\|_{\text {op }}\right) \\
& +\left(\delta_{1}(N)+2 n \delta_{1}(N)\right)^{2} \\
& \rightarrow \delta_{1}(N)+2\left(\delta_{1}(N)+2 n \delta_{1}(N)\right) \text {, }
\end{aligned}
$$

to leading order, since $\left\|\tilde{w}_{j}\right\|_{\text {op }} \rightarrow 1$ and $\left|\sigma_{j}\right| \rightarrow 1$ for $N \rightarrow \infty$. We set $\delta_{2}(N):=3 \delta_{1}(N)+4 n \delta_{1}(N)$ and insert $\bar{u}_{k}^{\prime}=\bar{u}_{k}^{*}, \bar{v}_{k}^{\prime}=$ $\mathcal{V} \bar{v}_{k}^{*}$ to obtain

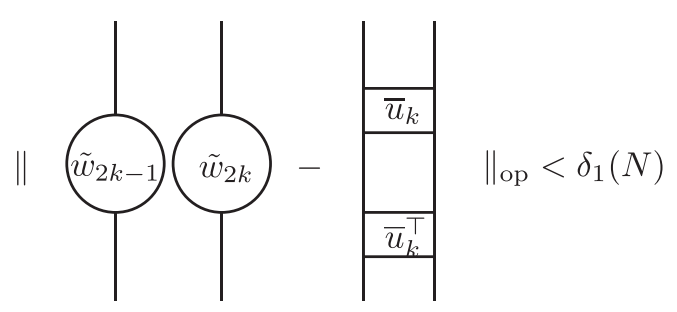

from Eq. (72) and

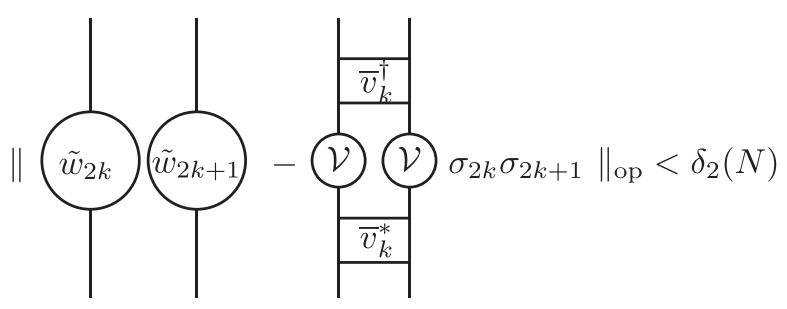

from Eq. (77). Setting $\gamma_{k}:=\sigma_{2 k} \sigma_{2 k+1}$ thus proves Eqs. (52) and (53). Equation (78) implies

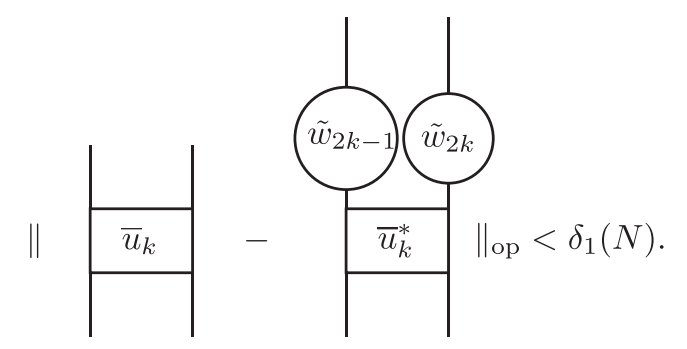

We insert Eq. (52) to obtain using Eq. (76)

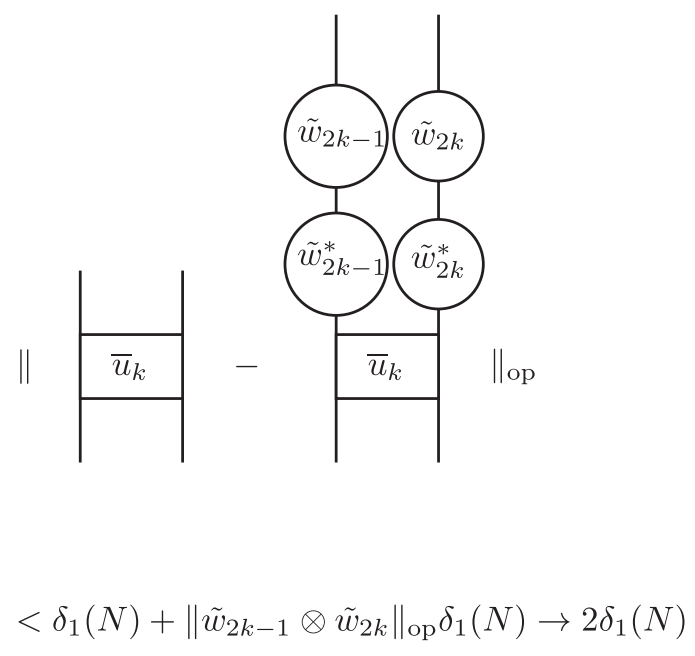

to leading order. We thus have

$$
\left\|\mathbb{1} \otimes \mathbb{1}-\left(\tilde{w}_{2 k-1} \tilde{w}_{2 k-1}^{*}\right) \otimes\left(\tilde{w}_{2 k} \tilde{w}_{2 k}^{*}\right)\right\|_{\mathrm{op}}<2 \delta_{1}(N),
$$

which implies

$$
\left\|\mathbb{1}-e^{i \tilde{\beta}_{k}} \tilde{w}_{2 k-1} \tilde{w}_{2 k-1}^{*}\right\|_{\mathrm{op}}<2 \delta_{1}(N)
$$

and

$$
\left\|\mathbb{1}-e^{-i \tilde{\beta}_{k}} \tilde{w}_{2 k} \tilde{w}_{2 k}^{*}\right\|_{\text {op }}<2 \delta_{1}(N)
$$

with $\tilde{\beta}_{k} \in \mathbb{C}$. In order to derive a bound on $\tilde{\beta}_{k}$, we need to bound the deviation of $\tilde{w}_{j}$ from a unitary matrix. If we apply Eq. (76) to

$$
\left\|\mathbb{1}-\bar{u}_{k} \bar{u}_{k}^{\top} \bar{u}_{k}^{*} \bar{u}_{k}^{\dagger}\right\|_{\text {op }}=\mathbb{1},
$$

Eq. (78) implies

$$
\begin{aligned}
& \left\|\mathbb{1}-\left(\tilde{w}_{2 k-1} \otimes \tilde{w}_{2 k}\right)\left(\tilde{w}_{2 k-1}^{\dagger} \otimes \tilde{w}_{2 k}^{\dagger}\right)\right\|_{\text {op }} \\
& \quad=\left\|\mathbb{1}-\left(\tilde{w}_{2 k-1} \tilde{w}_{2 k-1}^{\dagger}\right) \otimes\left(\tilde{w}_{2 k} \tilde{w}_{2 k}^{\dagger}\right)\right\|_{\text {op }}<2 \delta_{1}(N) .
\end{aligned}
$$

This in turn implies

$$
\begin{gathered}
\left\|\mathbb{1}-\tilde{w}_{2 k-1} \tilde{w}_{2 k-1}^{\dagger} \gamma_{k}\right\|_{\mathrm{op}}<2 \delta_{1}(N), \\
\left\|\mathbb{1}-\tilde{w}_{2 k} \tilde{w}_{2 k}^{\dagger} \frac{1}{\gamma_{k}}\right\|_{\mathrm{op}}<2 \delta_{1}(N)
\end{gathered}
$$


with $\gamma_{k} \in \mathbb{C}$. Based on these relations, we can bound (using the triangular inequality)

$$
\begin{aligned}
\left\|\tilde{w}_{2 k-1}\right\|_{\mathrm{op}}^{2}\left|\gamma_{k}\right| & =\left\|\tilde{w}_{2 k-1} \tilde{w}_{2 k-1}^{\dagger} \gamma_{k}\right\|_{\mathrm{op}} \\
& \leqslant\left\|-\mathbb{1}+\tilde{w}_{2 k-1} \tilde{w}_{2 k-1}^{\dagger} \gamma_{k}\right\|_{\mathrm{op}}+\|\mathbb{1}\|_{\mathrm{op}} \\
& <1+2 \delta_{1}(N)
\end{aligned}
$$

and

$$
\begin{aligned}
\left\|\tilde{w}_{2 k}\right\|_{\mathrm{op}}^{2} \frac{1}{\left|\gamma_{k}\right|} & =\left\|\tilde{w}_{2 k} \tilde{w}_{2 k}^{\dagger} \frac{1}{\gamma_{k}}\right\|_{\mathrm{op}} \\
& \leqslant\left\|-\mathbb{1}+\tilde{w}_{2 k} \tilde{w}_{2 k}^{\dagger} \frac{1}{\gamma_{k}}\right\|_{\mathrm{op}}+\|\mathbb{1}\|_{\mathrm{op}} \\
& <1+2 \delta_{1}(N) .
\end{aligned}
$$

With $\|1-\| \tilde{w}_{j} \|_{\text {op }} \mid<\frac{1}{2} \delta_{1}(N)$, Eqs. (89) and (90) give to leading order

$$
1-3 \delta_{1}(N)<\left|\gamma_{k}\right|<1+3 \delta_{1}(N),
$$

i.e., $|1-| \gamma_{k}||<3 \delta_{1}(N)$. Furthermore, Eq. (89) implies

$$
\begin{aligned}
& \left\|\tilde{w}_{2 k-1} \tilde{w}_{2 k-1}^{\dagger} \frac{\gamma_{k}-\gamma_{k}^{*}}{2}\right\|_{\text {op }} \\
& \leqslant \frac{1}{2}\left\|\mathbb{1}-\tilde{w}_{2 k-1} \tilde{w}_{2 k-1}^{\dagger} \gamma_{k}\right\|_{\mathrm{op}}+\frac{1}{2}\left\|\mathbb{1}-\tilde{w}_{2 k-1} \tilde{w}_{2 k-1}^{\dagger} \gamma_{k}^{*}\right\|_{\mathrm{op}} \\
& \quad<2 \delta_{1}(N),
\end{aligned}
$$

i.e., we have to leading order $\left|\operatorname{Im}\left(\gamma_{k}\right)\right|<2 \delta_{1}(N)$ and thus $|1-| \operatorname{Re}\left(\gamma_{k}\right)||<3 \delta_{1}(N)$. This together with Eq. (87) and $\tilde{w}_{2 k-1} \tilde{w}_{2 k-1}^{\dagger} \geqslant 0$ results in

$$
\left|1-\operatorname{Re}\left(\gamma_{k}\right)\right|<3 \delta_{1}(N) .
$$

Hence, we arrive at

$$
\begin{aligned}
\| \mathbb{1} & -\tilde{w}_{2 k-1} \tilde{w}_{2 k-1}^{\dagger} \|_{\text {op }} \\
& \leqslant\left\|\mathbb{1}-\tilde{w}_{2 k-1} \tilde{w}_{2 k-1}^{\dagger} \gamma_{k}\right\|_{\text {op }}+\left\|\tilde{w}_{2 k-1} \tilde{w}_{2 k-1}^{\dagger}\left(\gamma_{k}-1\right)\right\|_{\mathrm{op}} \\
& <2 \delta_{1}(N)+\left\|\tilde{w}_{2 k-1}\right\|_{\mathrm{op}}^{2}\left|\gamma_{k}-1\right| \\
& <4 \delta_{1}(N)+\left\|\tilde{w}_{2 k-1}\right\|_{\mathrm{op}}^{2}\left|\operatorname{Re}\left(\gamma_{k}\right)-1\right|<7 \delta_{1}(N)
\end{aligned}
$$

and

$$
\begin{aligned}
& \| \mathbb{1}-\tilde{w}_{2 k} \tilde{w}_{2 k}^{\dagger} \|_{\text {op }} \\
& \leqslant\left\|\mathbb{1}-\tilde{w}_{2 k} \tilde{w}_{2 k}^{\dagger} \frac{1}{\gamma_{k}}\right\|_{\text {op }}+\left\|\tilde{w}_{2 k} \tilde{w}_{2 k}^{\dagger}\left(\frac{1}{\gamma_{k}}-1\right)\right\|_{\text {op }} \\
&< 2 \delta_{1}(N)+\left\|\tilde{w}_{2 k}\right\|_{\text {op }}^{2} \frac{1}{\left|\gamma_{k}\right|}\left|1-\gamma_{k}\right| \\
&<4 \delta_{1}(N)+\frac{\left\|\tilde{w}_{2 k}\right\|_{\text {op }}^{2}}{\left|\gamma_{k}\right|}\left|\operatorname{Re}\left(\gamma_{k}\right)-1\right|<7 \delta_{1}(N) .
\end{aligned}
$$

Equations (94) and (95) taken together are

$$
\left\|\mathbb{1}-\tilde{w}_{j} \tilde{w}_{j}^{\dagger}\right\|_{\text {op }}<7 \delta_{1}(N)
$$

and thus bound the extend to which $\tilde{w}_{j}$ deviates from a unitary matrix. We now proceed to derive a bound on $\tilde{\beta}_{k}$. Equation (83) implies (since $\left\|\tilde{w}_{j}\right\|_{\text {op }} \rightarrow 1$ for $N \rightarrow \infty$ )

$$
\left\|\tilde{w}_{2 k-1}^{\top}-\tilde{w}_{2 k-1} \tilde{w}_{2 k-1}^{*} \tilde{w}_{2 k-1}^{\top} e^{i \tilde{\beta}_{k}}\right\|_{\mathrm{op}}<2 \delta_{1}(N) .
$$

Consequently, Eq. (96) yields by virtue of Eq. (76)

$$
\left\|\tilde{w}_{2 k-1}^{\top}-\tilde{w}_{2 k-1} e^{i \tilde{\beta}_{k}}\right\|_{\mathrm{op}}<9 \delta_{1}(N) .
$$

We take the transpose of this relation and use Eq. (76) again to obtain

$$
\left\|w_{2 k-1}^{\top}-w_{2 k-1}^{\top} e^{2 i \tilde{\beta}_{k}}\right\|<18 \delta_{1}(N) .
$$

To leading order, this implies

$$
\left|1-e^{2 i \tilde{\beta}_{k}}\right|<18 \delta_{1}(N) .
$$

Hence, if we define

$$
\pm 1-e^{i \tilde{\beta}_{k}}=d_{k},
$$

it has to hold to leading order that

$$
\left|d_{k}\right|<9 \delta_{1}(N) .
$$

Using the last two relations and Eq. (83) results with Eq. (76) in

$$
\left\|\mathbb{1} \mp \tilde{w}_{2 k-1} \tilde{w}_{2 k-1}^{*}\right\|_{\text {op }}<11 \delta_{1}(N) .
$$

We obtain again a topological index up to corrections which vanish in the thermodynamic limit. Using Eq. (84), one gets similarly

$$
\left\|\mathbb{1} \mp \tilde{w}_{2 k} \tilde{w}_{2 k}^{*}\right\|_{\text {op }}<11 \delta_{1}(N)
$$

with the same sign; however the sign of the pair $(2 k-1,2 k)$ might differ from the one of $(2 k+1,2 k+2)$. To prove that they are the same, note that Eq. (79) implies

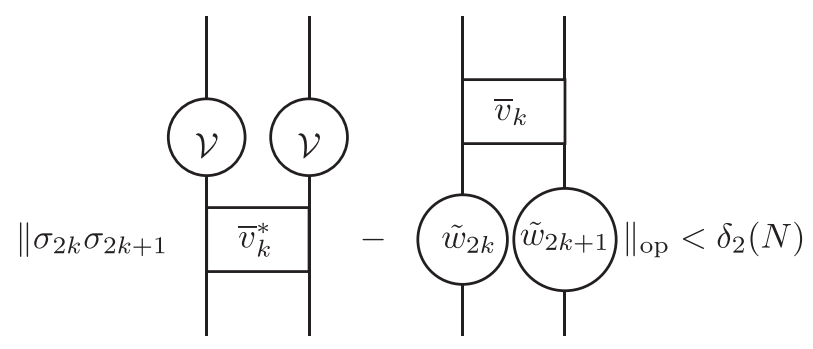

and

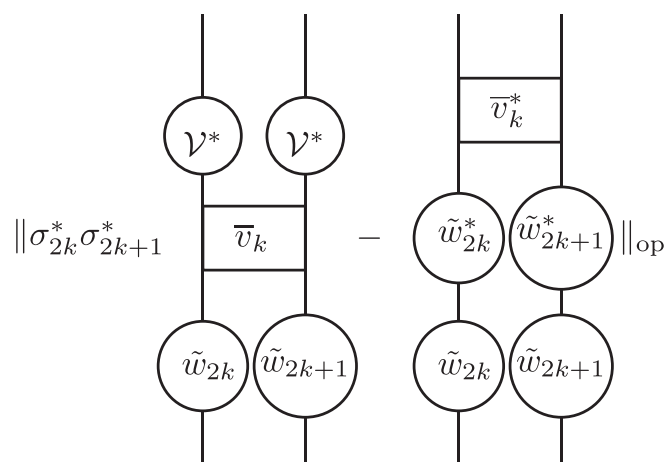


In the following expression we use Eq. (76) and bound the left term with Eq. (105) and the right term with Eq. (106):
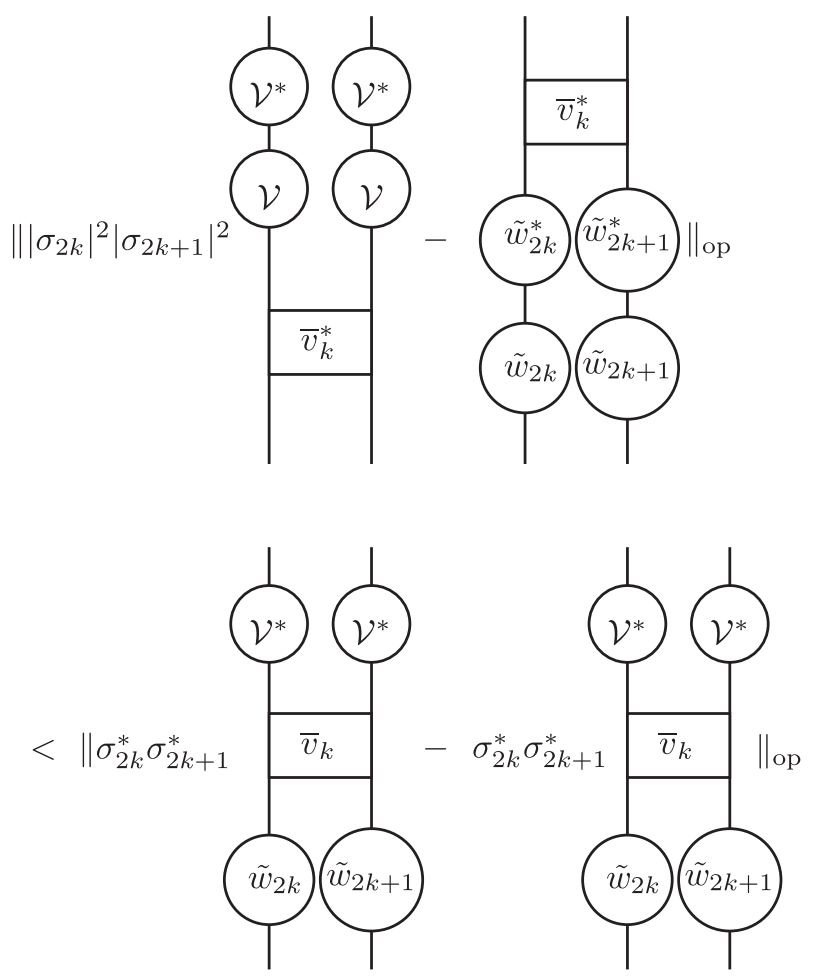

$+\delta_{2}(N)\left\|\tilde{w}_{2 k} \otimes \tilde{w}_{2 k+1}\right\|_{\text {op }}+\delta_{2}(N)\left|\sigma_{2 k}\right|\left|\sigma_{2 k+1}\right|$

$\rightarrow 2 \delta_{2}(N)$

to leading order. Owing to $\mathcal{V} \mathcal{V}^{*}= \pm \mathbb{1}$ we therefore have

$$
\begin{aligned}
& \left\|\left.|| \sigma_{2 k}\right|^{2}\left|\sigma_{2 k+1}\right|^{2} \mathbb{1}-\left(\tilde{w}_{2 k}^{*} \tilde{w}_{2 k}\right) \otimes\left(\tilde{w}_{2 k+1}^{*} \tilde{w}_{2 k+1}\right)\right\|_{\text {op }} \\
& \quad<2 \delta_{2}(N) .
\end{aligned}
$$

Hence, Eqs. (76), (83), and (84) yield

$$
\left.|| \sigma_{2 k}\right|^{2}\left|\sigma_{2 k+1}\right|^{2}-e^{i \tilde{\beta}_{k}-i \tilde{\beta}_{k+1}} \mid<4 \delta_{1}(N)+2 \delta_{2}(N) .
$$

Now we insert Eq. (101),

$$
\left.|| \sigma_{2 k}\right|^{2}\left|\sigma_{2 k+1}\right|^{2}-\frac{ \pm 1-d_{k}}{ \pm 1-d_{k+1}} \mid<4 \delta_{1}(N)+2 \delta_{2}(N),
$$

which shows that the signs in Eqs. (103) and (104) have to be identical for all $k$ for sufficiently large $N$. We are thus left with

$$
\left\|\mathbb{1} \mp \tilde{w}_{j} \tilde{w}_{j}^{*}\right\|_{\text {op }}<11 \delta_{1}(N)
$$

with identical sign for all $j=1,2, \ldots, 2 n$.

The entanglement spectrum is given by the "entanglement energies," which are the eigenvalues of the entanglement Hamiltonian $H_{\text {ent }}$ defined by $\rho_{L}=e^{-H_{\text {ent }}} . \rho_{L}$ is the reduced density matrix obtained after tracing out half of the chain from a certain eigenstate.

The proof of Statement 1 is concluded by showing the following:
Lemma 4. If the topological index is -1 , i.e., $\| \mathbb{1}+$ $\tilde{w}_{j} \tilde{w}_{j}^{*} \|_{\text {op }}<11 \delta_{1}(N)$, the eigenvalues of the half-chain reduced density matrix $\rho_{L}$ of any eigenstate are fourfold degenerate up to corrections of order $\delta_{1}(N)$.

Note that the fact that the negative sign corresponds to the topologically nontrivial phase can be seen by drawing the analogy to symmetry-protected topological ground states [84]. Alternatively, one may investigate the system described by the Hamiltonian (1) with $\sigma_{h}=\sigma_{V}=0$, which can be diagonalized exactly by a translationally invariant quantum circuit [94] with $\ell=2$ and $w_{j} w_{j}^{*}=-\mathbb{1}$, see Appendix A.

Proof of Lemma 4. We trace out sites $\frac{N}{2}+\frac{3}{2} \ell+1, \frac{N}{2}+$ $\frac{3}{2} \ell+2, \ldots, N, 1,2, \ldots, \frac{3}{2} \ell$ for a certain approximate eigenstate given by 1 -bits $\left(l_{1}, l_{2}, \ldots, l_{N / 2}\right)=\left(\bar{l}_{1}, \bar{l}_{2}, \ldots, \bar{l}_{n / 2}\right)$. The resulting reduced density matrix $\rho_{L}$ can be expressed in terms of virtual reduced density matrices $\sigma_{L}$ and $\sigma_{R}$, which we define as follows:

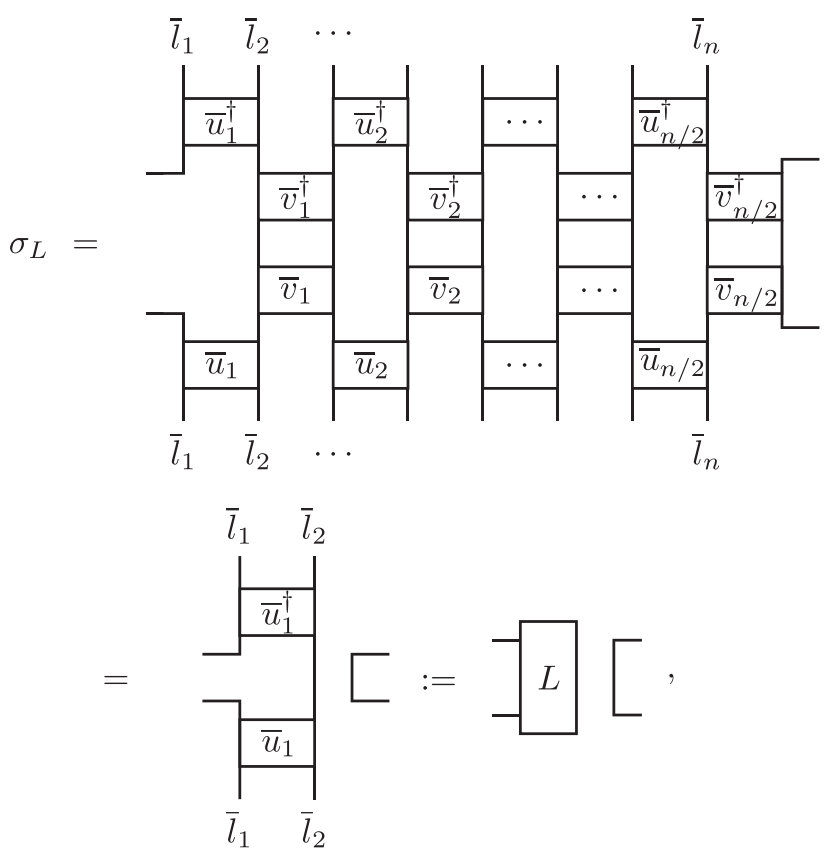

where we canceled unitaries with their adjoints and used the fact that the upper and lower dangling legs have identical indices. We obtain a similar relation for $\sigma_{R}$ defined for the other half of the system,

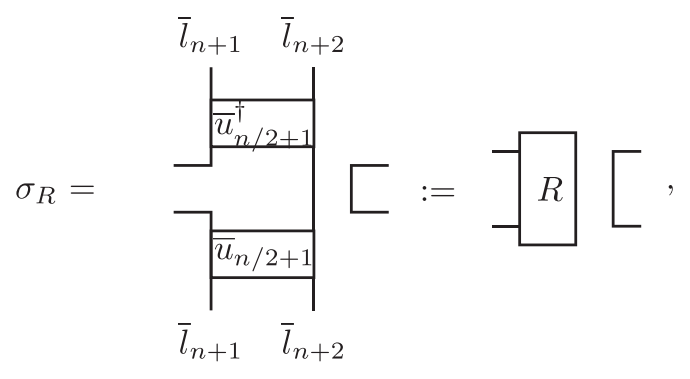


We now derive a relation between $\rho_{L}$ and $\sigma_{L}, \sigma_{R}$, which is a special case of the bulk-boundary correspondence [95]. To that end, we employ the MPO representation Eqs. (16) and (17). Let $\mathcal{A}$ be the tensor corresponding to a concatenation of tensors $A_{1}, A_{2}, \ldots, A_{n / 2}$ with fixed 1-bits $l_{1}, l_{2}, \ldots, l_{N / 2}$ (lower indices)

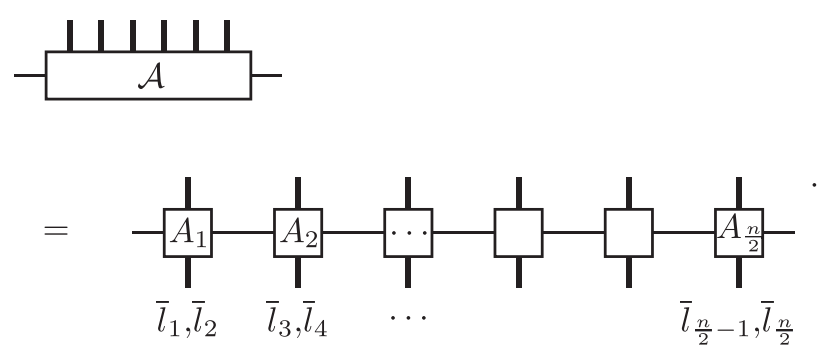

We combine all physical indices (vertical legs) of $\mathcal{A}$ to an index $a$ and all virtual indices (horizontal legs) to $\alpha$. We define $\mathcal{B}$ similarly for the other half of the chain (with physical index $b$ ). The reduced density matrix is thus

$$
\left[\rho_{L}\right]_{a a^{\prime}}=\sum_{b, \alpha, \beta} \mathcal{A}_{a \alpha} \mathcal{B}_{\alpha b} \mathcal{B}_{\beta b}^{*} \mathcal{A}_{a^{\prime} \beta}^{*}
$$

In matrix representation, $\rho_{L}=\mathcal{A B B}^{\dagger} \mathcal{A}^{\dagger}$. Using Sylvester's determinant theorem, it is easy to show that for any complex $m \times n$ matrix $R$ and $n \times m$ matrix $S$, $\operatorname{spec}(R S)=\operatorname{spec}(S R)$ up to zero eigenvalues. That is, the two spectra are identical, and only the number of zero eigenvalues can change upon commuting the matrices (for $m=n$ the spectra are thus completely identical). We will ignore such zero eigenvalues henceforth. (They correspond to infinite entanglement energies.) Now we obtain $\operatorname{spec}\left(\rho_{L}\right)=\operatorname{spec}\left(\mathcal{A B B}^{\dagger} \mathcal{A}^{\dagger}\right)=\operatorname{spec}\left(\mathcal{B B}^{\dagger} \mathcal{A}^{\dagger} \mathcal{A}\right)=$ $\operatorname{spec}\left(\sigma_{R}^{\top} \sigma_{L}\right)$ since $\sigma_{L}=\mathcal{A}^{\dagger} \mathcal{A}, \sigma_{R}=\left(\mathcal{B B}^{\dagger}\right)^{\top}$. Notice that in the product $\mathcal{B B}^{\dagger} \mathcal{A}^{\dagger} \mathcal{A}$ the conjugate matrices of the left and right block are multiplied, i.e., the upper horizontal legs have to be connected: $\sigma:=\sigma_{R}^{\top} \sigma_{L}$ can thus be written as

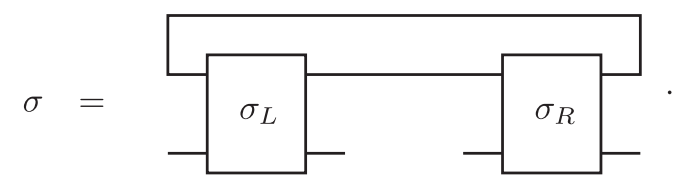

We finally obtain that the entanglement spectrum is given by $\operatorname{spec}\left(H_{\text {ent }}\right)=\operatorname{spec}[-\ln (\sigma)]$ up to varying numbers of entanglement energies at $+\infty$. We insert Eqs. (112) and (113) into the last relation and obtain

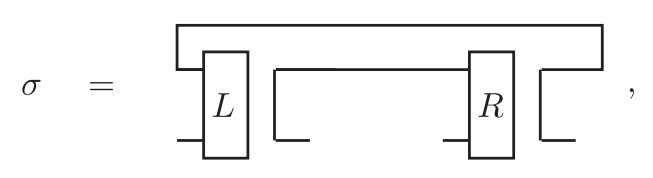

which in algebraic notation is simply $\sigma=L \otimes R$. We show that the spectrum of $L$ is twofold degenerate up to $\delta_{1}(N)$ corrections. The same line of reasoning demonstrates twofold degeneracy of the spectrum of $R$ and thus fourfold degeneracy of the entanglement spectrum.

Let us define

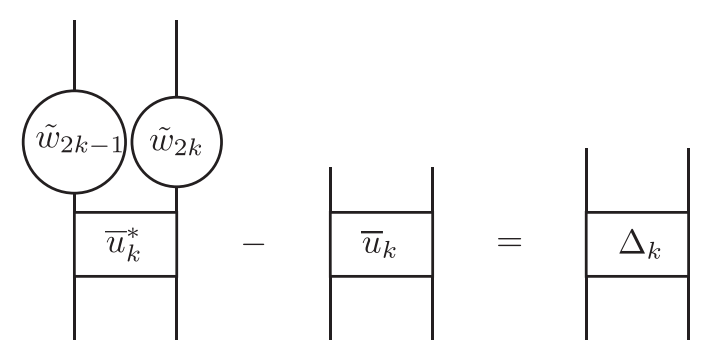

Equation (80) implies $\left\|\Delta_{k}\right\|_{\text {op }}<\delta_{1}(N)$. By virtue of Eq. (118), we have

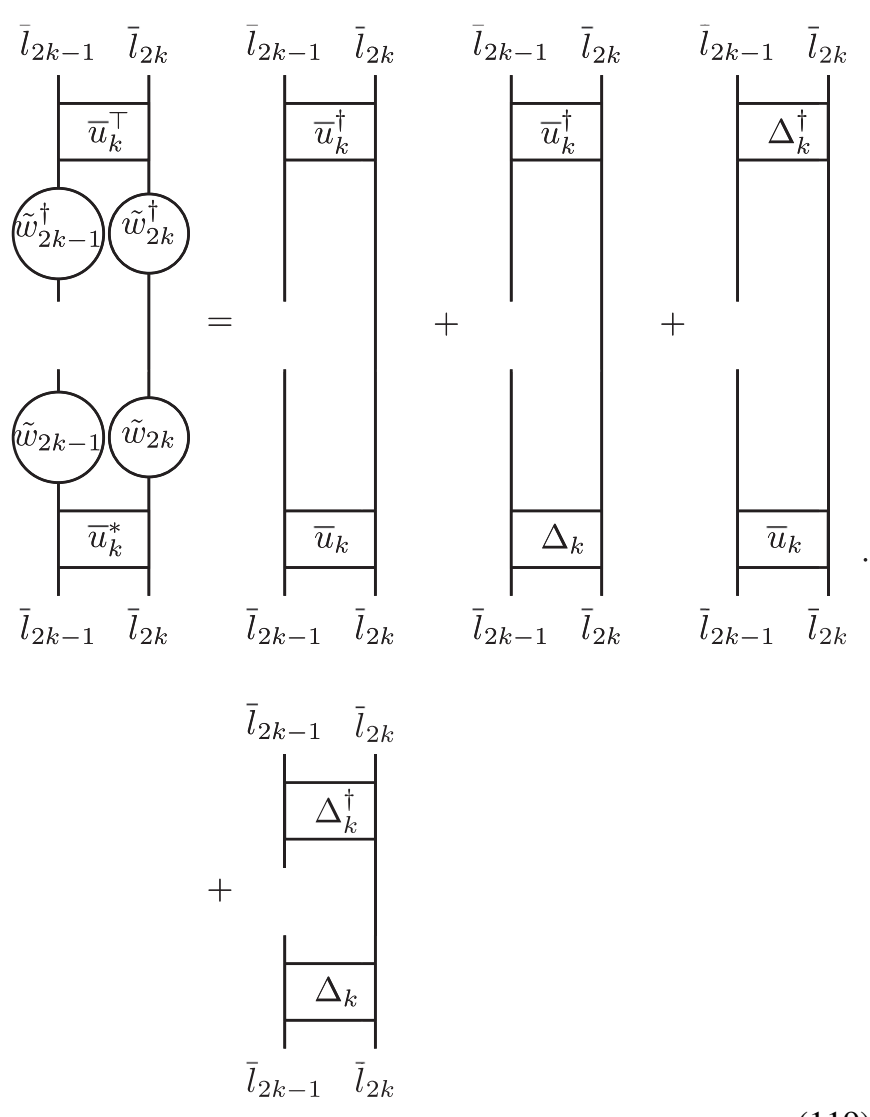

(119) 
Using Eq. (96), we obtain for $D_{j}:=\mathbb{1}-\tilde{w}_{j} \tilde{w}_{j}^{\dagger}$ that $\left\|D_{j}\right\|_{\text {op }}<$ $7 \delta_{1}(N)$. We thus have

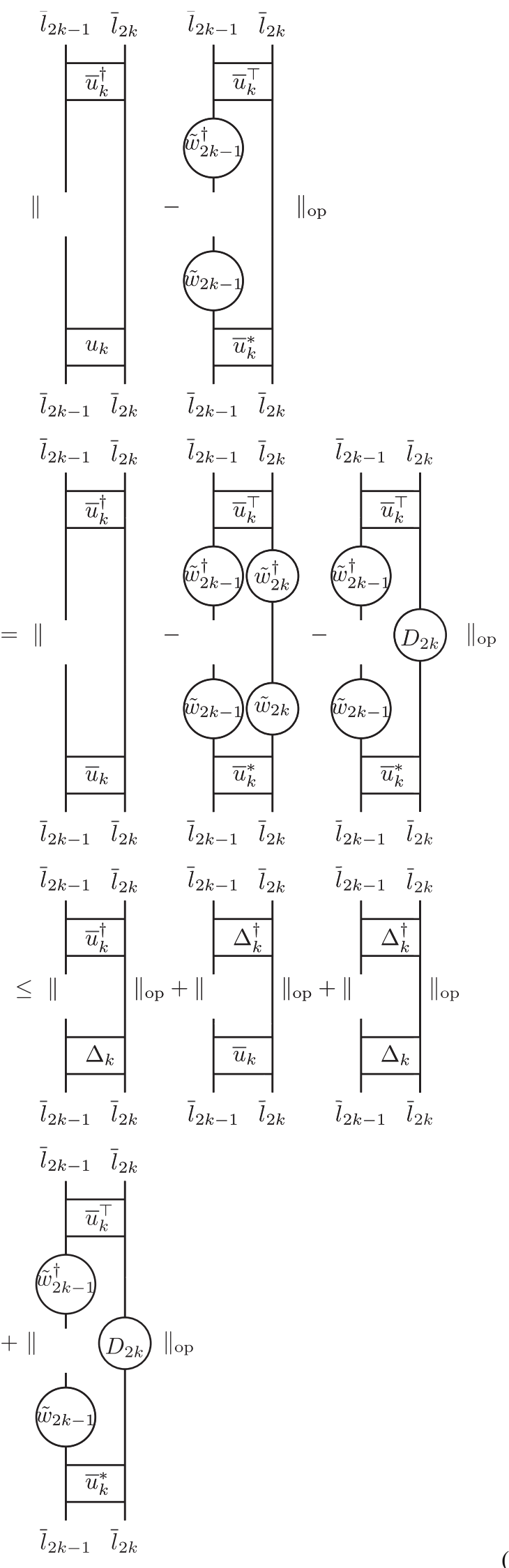

where the last inequality follows from Eq. (118). We can bound the terms in the last expression of Eq. (120) using the property

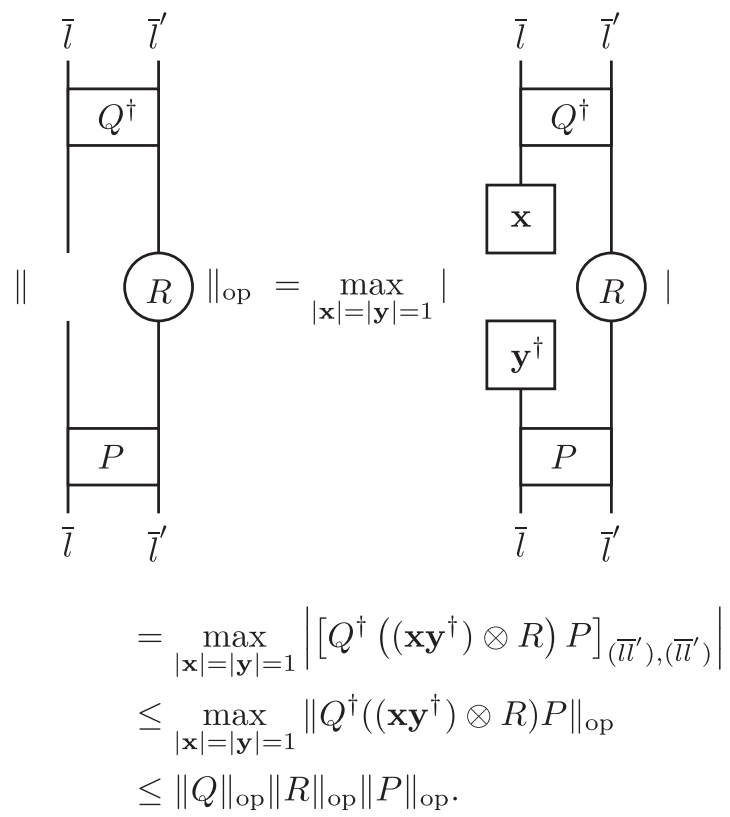

Therefore, the final expression of Eq. (120) fulfills

$$
\begin{aligned}
(120) & <2\left\|\Delta_{k}\right\|_{\text {op }}+\left\|\Delta_{k}\right\|_{\text {op }}^{2}+\left\|D_{2 k}\right\|_{\text {op }} \\
& <2 \delta_{1}(N)+\delta_{1}^{2}(N)+7 \delta_{1}(N) \rightarrow 9 \delta_{1}(N)
\end{aligned}
$$

due to $\left\|D_{j}\right\|_{\text {op }}<7 \delta_{1}(N)$. Owing to the definition Eq. (112) of $L$, the inequalities (120) and (122) imply

$$
\left\|L-\tilde{w}_{2 n-1}^{\dagger} L^{*} w_{2 n-1}\right\|_{\text {op }}<9 \delta_{1}(N) .
$$

From that we obtain

$$
\begin{aligned}
& \left\|\tilde{w}_{2 n-1}^{\dagger} L^{*} \tilde{w}_{2 n-1} \tilde{w}_{2 n}^{\dagger}-L \tilde{w}_{2 n-1}^{\dagger}\right\|_{\text {op }} \\
& \quad<9 \delta_{1}(N)\left\|\tilde{w}_{2 n-1}\right\|_{\text {op }} \rightarrow 9 \delta_{1}(N) .
\end{aligned}
$$

Due to $\left\|\mathbb{1}-\tilde{w}_{j} \tilde{w}_{j}^{\dagger}\right\|_{\text {op }}<7 \delta_{1}(N)$ and Eq. (76), we thus arrive at

$$
\begin{aligned}
& \left\|\tilde{w}_{2 n-1}^{\dagger} L^{*}-L \tilde{w}_{2 n-1}^{\dagger}\right\|_{\mathrm{op}} \\
& \quad<9 \delta_{1}(N)+7 \delta_{1}(N)\|L\|_{\mathrm{op}}\left\|\tilde{w}_{2 n-1}^{\dagger}\right\|_{\mathrm{op}} \\
& \quad \rightarrow 9 \delta_{1}(N)+7 \delta_{1}(N)\|L\|_{\mathrm{op}} \leqslant 16 \delta_{1}(N),
\end{aligned}
$$

where the last inequality follows from Eq. (121).

Since $L$ is Hermitian, there exists an orthonormal set of eigenvectors. We group them into matrices $T_{s}$ spanning linear subspaces with the same eigenvalue $\mu_{s}\left(0 \leqslant \mu_{s} \leqslant 1\right)$, i.e.,

$$
L T_{s}=\mu_{s} T_{s}
$$

and $L^{*} T_{s}^{*}=\mu_{s} T_{s}^{*}$. Defining $\tilde{D}:=\tilde{w}_{2 n-1}^{\dagger} L^{*}-L \tilde{w}_{2 n-1}^{\dagger}$ [i.e., $\left.\|\tilde{D}\|_{\text {op }}<16 \delta_{1}(N)\right]$, we thus obtain

$$
\mu_{s} \tilde{w}_{2 n-1}^{\dagger} T_{s}^{*}=L \tilde{w}_{2 n-1}^{\dagger} T_{s}^{*}+\tilde{D} T_{s}^{*}
$$

We can decompose

$$
\tilde{w}_{2 n-1}^{\dagger} T_{s}^{*}=T_{s} A_{s}+\sum_{s^{\prime} \neq s} T_{s^{\prime}} A_{s^{\prime}}
$$


with matrices $A_{s^{\prime}}$ of size $\operatorname{dim}\left(T_{s^{\prime}}\right) \times \operatorname{dim}\left(T_{s}\right)$. As a result, Eq. (127) yields

$$
\sum_{s^{\prime} \neq s}\left(\mu_{s^{\prime}}-\mu_{s}\right) T_{s^{\prime}} A_{s^{\prime}}=-\tilde{D} T_{s}^{*}
$$

Since the $T_{s}, T_{s^{\prime}}$ are all linearly independent and of operator norm 1 , we obtain

$$
\begin{aligned}
\|\tilde{D}\|_{\mathrm{op}} & =\left\|\sum_{s^{\prime} \neq s}\left(\mu_{s^{\prime}}-\mu_{s}\right) T_{s^{\prime}} A_{s^{\prime}}\right\|_{\mathrm{op}} \\
& =\max _{|\mathbf{v}|=1}\left|\sum_{s^{\prime} \neq s}\left(\mu_{s^{\prime}}-\mu_{s}\right) T_{s^{\prime}} A_{s^{\prime}} \mathbf{v}\right| \\
& =\max _{|\mathbf{v}|=1} \sqrt{\sum_{s^{\prime} \neq s}\left|\left(\mu_{s^{\prime}}-\mu_{s}\right) T_{s^{\prime}} A_{s^{\prime}} \mathbf{v}\right|^{2}} \\
& \geqslant \sqrt{\sum_{s^{\prime} \neq s}\left|\left(\mu_{s^{\prime}}-\mu_{s}\right) T_{s^{\prime}} A_{s^{\prime}} \mathbf{w}_{1}\right|^{2}},
\end{aligned}
$$

where $\mathbf{w}_{1}$ is a unit vector where the function $f(\mathbf{w})=$ $\sqrt{\sum_{s^{\prime} \neq s}\left|T_{s^{\prime}} A_{s^{\prime}} \mathbf{w}\right|^{2}}$ is maximal. Hence, we can further lower bound the final term of Eq. (130) by

$$
\begin{aligned}
(130) & \geqslant\left(\min _{s^{\prime \prime} \neq s}\left|\mu_{s^{\prime \prime}}-\mu_{s}\right|\right) \max _{|\mathbf{w}|=1} \sqrt{\sum_{s^{\prime} \neq s}\left|T_{s^{\prime}} A_{s^{\prime}} \mathbf{w}\right|^{2}} \\
& =\left(\min _{s^{\prime \prime} \neq s}\left|\mu_{s^{\prime \prime}}-\mu_{s}\right|\right)\left\|\sum_{s^{\prime} \neq s} T_{s^{\prime}} A_{s^{\prime}}\right\|_{\text {op }} .
\end{aligned}
$$

This results in

$$
\begin{aligned}
\left\|\sum_{s^{\prime} \neq s} T_{s^{\prime}} A_{s^{\prime}}\right\|_{\text {op }} & \leqslant \frac{\|\tilde{D}\|_{\text {op }}}{\min _{s^{\prime \prime} \neq s}\left|\mu_{s^{\prime \prime}}-\mu_{s}\right|} \\
& <\frac{16 \delta_{1}(N)}{\min _{s^{\prime \prime} \neq s}\left|\mu_{s^{\prime \prime}}-\mu_{s}\right|} .
\end{aligned}
$$

Equation (128) implies thus

$$
\left\|\tilde{w}_{2 n-1}^{\dagger} T_{s}^{*}-T_{s} A_{s}\right\|_{\mathrm{op}}<\frac{16 \delta_{1}(N)}{\min _{s^{\prime} \neq s}\left|\mu_{s^{\prime}}-\mu_{s}\right|}
$$

and

$$
\left\|\tilde{w}_{2 n-1}^{\top} T_{s}-T_{s}^{*} A_{s}^{*}\right\|_{\mathrm{op}}<\frac{16 \delta_{1}(N)}{\min _{s^{\prime} \neq s}\left|\mu_{s^{\prime}}-\mu_{s}\right|} .
$$

After multiplying Eq. (133) from the left by $\tilde{w}_{2 n-1}^{\top}$, Eqs. (76) and (111) (with the negative topological index) result in

$$
\left\|T_{s}^{*}+\tilde{w}_{2 n-1}^{\top} T_{s} A_{s}\right\|_{\mathrm{op}}<\frac{16 \delta_{1}(N)\left\|\tilde{w}_{2 n-1}\right\|_{\mathrm{op}}}{\min _{s^{\prime \prime} \neq s}\left|\mu_{s^{\prime}}-\mu_{s}\right|}+11 \delta_{1}(N) .
$$

The last two inequalities yield by way of Eq. (76)

$$
\begin{aligned}
& \left\|T_{s}^{*}+T_{s}^{*} A_{s}^{*} A_{s}\right\|_{\mathrm{op}} \\
& \quad<\frac{16 \delta_{1}(N)\left(\left\|\tilde{w}_{2 n-1}\right\|_{\mathrm{op}}+\left\|A_{s}\right\|_{\mathrm{op}}\right)}{\min _{s^{\prime} \neq s}\left|\mu_{s^{\prime}}-\mu_{s}\right|}+11 \delta_{1}(N) .
\end{aligned}
$$

Since to zeroth order $\tilde{w}_{2 n-1}^{\dagger}$ is unitary, Eq. (128) and orthonormality of the $T_{s}, T_{s^{\prime}}$ implies $\left\|A_{s}\right\|_{\text {op }} \leqslant 1$ to zeroth order, i.e., for $Z:=\mathbb{1}+A_{s}^{*} A_{s}$ we have to leading order

$$
\|Z\|_{\mathrm{op}}<\frac{32 \delta_{1}(N)}{\min _{s^{\prime} \neq s}\left|\mu_{s^{\prime}}-\mu_{s}\right|}+11 \delta_{1}(N)
$$

Hence, since $\left|\operatorname{det}\left(A_{s}\right)\right|^{2}=\operatorname{det}\left(A_{s}^{*} A_{s}\right)=\operatorname{det}(-\mathbb{1}+Z)$, the degeneracy $\operatorname{dim}\left(A_{s}\right)$ of $\mu_{s}$ cannot be odd, otherwise $\operatorname{det}(-\mathbb{1}+Z)$ would become negative for sufficiently large $N$ (where the $\mu_{s}$ converge to the exact eigenvalues).

Note that for finite $N$, such even degenerate multiplets can thus have a splitting $\left|\mu_{s^{\prime}}-\mu_{s}\right|$, which is at most of order $\delta_{1}(N)=(2+4 N) \delta(N)=2^{13 / 4}(1+2 N) \sqrt{\frac{c N}{3}} e^{-\frac{\alpha N^{\mu}}{2 c^{\prime}}}$.

This concludes the proof of Statement 1.

\section{Proof of Statement 2}

We assume $N$ to be finite, and strict topological protection will again follow in the limit $N \rightarrow \infty$. Suppose the adiabatic perturbation is described by a parameter $\lambda \in[0,1]$ with corresponding Hamiltonian $H(\lambda)$ such that Conditions 1 to 3 of the Theorem are fulfilled for all $\lambda$. Condition 1 thus requires the existence of a unitary $U(\lambda)$ diagonalizing the Hamiltonian such that $\tau_{i}^{z}(\lambda)=U(\lambda) \sigma_{i}^{z} U^{\dagger}(\lambda)$ fulfills $\left\|\tilde{\tau}_{i}^{z}(\lambda)-\tau_{i}^{z}(\lambda)\right\|_{\text {op }}<$ $c(\lambda) e^{-\frac{\ell}{\xi_{i}(\lambda)}}$ with $\tilde{\tau}_{i}^{z}(\lambda)=\tilde{U}(\lambda) \sigma_{i}^{z} \tilde{U}^{\dagger}(\lambda)=\bar{U}(\lambda) \sigma_{i}^{z} \bar{U}^{\dagger}(\lambda)$. Due to Condition 3, we can assume that $H(\lambda)$ (at least after some infinitely small $\lambda$-independent perturbation $\epsilon V$ ) is nondegenerate for almost all $\lambda$. Degeneracies only appear at level crossings, which are isolated points for finite $N$.

First of all, note that one can always define a unitary $U_{\text {cont }}(\lambda)$ which diagonalizes the overall Hamiltonian $H(\lambda)$ and changes continuously as a function of $\lambda$. This can be seen by comparing the two limits $\lim _{\epsilon \rightarrow 0 \pm} H(\lambda+\epsilon)$ expressed in terms of $U_{\text {cont }}(\lambda+\epsilon)$ and $E(\lambda+\epsilon)$. For almost all $\lambda, U_{\text {cont }}(\lambda)$ has to be related to $U(\lambda)$ via

$$
U(\lambda)=U_{\text {cont }}(\lambda) P(\lambda)
$$

where $P(\lambda)$ is a permutation matrix whose nonvanishing elements have arbitrary phases (and magnitude 1). According to Lemmas 1 and 2 we thus have

$$
\left\|U_{\text {cont }}(\lambda) P(\lambda)-\bar{U}(\lambda)\right\|_{\text {op }}<\delta_{1}(N) .
$$

Now consider two points $\lambda_{1}, \lambda_{2} \in[0,1]$. We want to show that the topological index of the corresponding quantum circuits $\bar{U}\left(\lambda_{1}\right), \bar{U}\left(\lambda_{2}\right)$ is the same. Due to the triangular inequality,

$$
\begin{aligned}
& \left\|\bar{U}\left(\lambda_{1}\right)-\bar{U}\left(\lambda_{2}\right) P^{\dagger}\left(\lambda_{2}\right) P\left(\lambda_{1}\right)\right\|_{\mathrm{op}} \\
& \leqslant\left\|\bar{U}\left(\lambda_{1}\right)-U_{\text {cont }}\left(\lambda_{1}\right) P\left(\lambda_{1}\right)\right\|_{\mathrm{op}} \\
& \quad+\left\|U_{\mathrm{cont}}\left(\lambda_{1}\right) P\left(\lambda_{1}\right)-U_{\mathrm{cont}}\left(\lambda_{2}\right) P\left(\lambda_{1}\right)\right\|_{\mathrm{op}} \\
& \quad+\left\|U_{\mathrm{cont}}\left(\lambda_{2}\right) P\left(\lambda_{1}\right)-\bar{U}\left(\lambda_{2}\right) P^{\dagger}\left(\lambda_{2}\right) P\left(\lambda_{1}\right)\right\|_{\mathrm{op}} \\
& \quad<2 \delta_{1}(N)+\left\|U_{\mathrm{cont}}\left(\lambda_{1}\right)-U_{\mathrm{cont}}\left(\lambda_{2}\right)\right\|_{\mathrm{op}}
\end{aligned}
$$


due to Eq. (139). We choose $\lambda_{2}:=\lambda_{1}+\epsilon$, such that because of the continuity of $U_{\text {cont }}(\lambda)$

$$
\left\|\bar{U}\left(\lambda_{1}\right)-\bar{U}\left(\lambda_{2}\right) P^{\dagger}\left(\lambda_{2}\right) P\left(\lambda_{1}\right)\right\|_{\mathrm{op}}<2 \delta_{1}(N)+O(\epsilon),
$$

where the $O(\epsilon)$ term is $N$ independent. We consider the quantum circuit defined by $\bar{U}_{21}:=\bar{U}^{\dagger}\left(\lambda_{2}\right) \bar{U}\left(\lambda_{1}\right)$, which is due to Eq. (141) close to a permutation matrix. That quantum circuit reads graphically [we denote the unitaries of $\bar{U}\left(\lambda_{1}\right)$ by $\bar{u}_{k}, \bar{v}_{k}$ and those of $\bar{U}\left(\lambda_{2}\right)$ by $\left.\hat{u}_{k}, \hat{v}_{k}\right]$

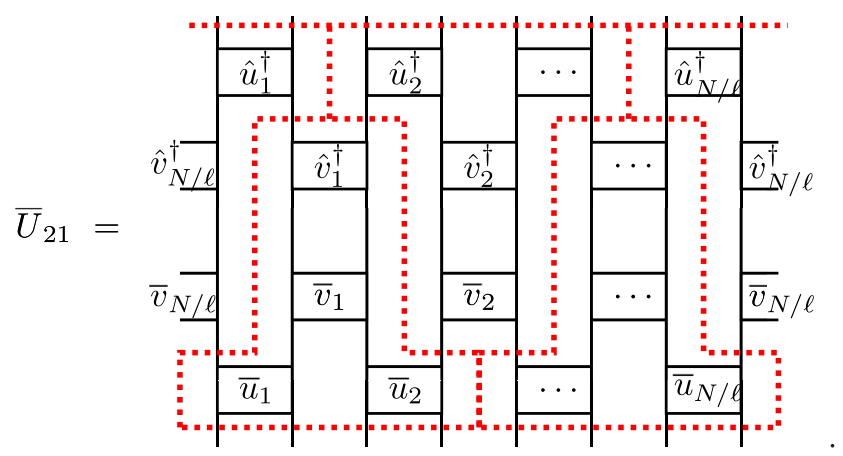

$\bar{U}_{21}$ can also be written as a two-layer quantum circuit if the unitaries are blocked together as indicated by red dashed lines. From Lemma 2 we know that $\left\|\bar{U}\left(\lambda_{1}\right)-\mathcal{V} \bar{U}^{*}\left(\lambda_{1}\right)\right\|_{\text {op }}<$ $\delta_{1}(N)$ and $\left\|\bar{U}\left(\lambda_{2}\right)-\mathcal{V} \bar{U}^{*}\left(\lambda_{2}\right)\right\|_{\text {op }}<\delta_{1}(N)$, which together with Eq. (76) implies

$$
\left\|\bar{U}_{21}-\bar{U}_{21}^{*}\right\|_{\mathrm{op}}<2 \delta_{1}(N)
$$

Hence, we can apply Lemma 3, which in this case states that

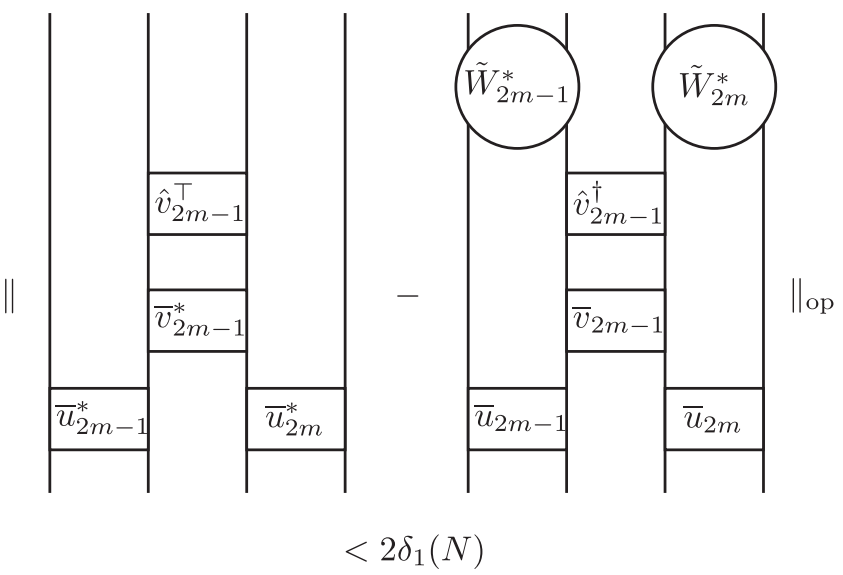

with $\left\|\mathbb{1} \mp \tilde{W}_{k} \tilde{W}_{k}^{*}\right\|_{\text {op }}<22 \delta_{1}(N)$. On the other hand, Lemma 3 applied to the individual quantum circuits $\bar{U}\left(\lambda_{1}\right), \bar{U}\left(\lambda_{2}\right)$ implies to leading order using Eq. (76) $\left[\hat{w}_{j}\right.$ for $\bar{U}\left(\lambda_{2}\right)$ corresponds to $\tilde{w}_{j}$ for $\left.\bar{U}\left(\lambda_{1}\right)\right]$

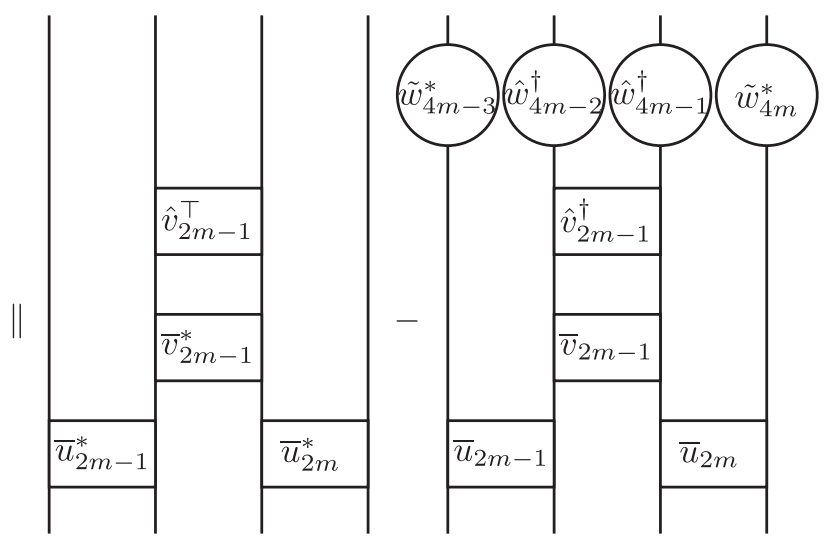

$\times \frac{\gamma_{2 m-1}^{*}}{\hat{\gamma}_{2 m-1}^{*}} \|_{\text {op }}$

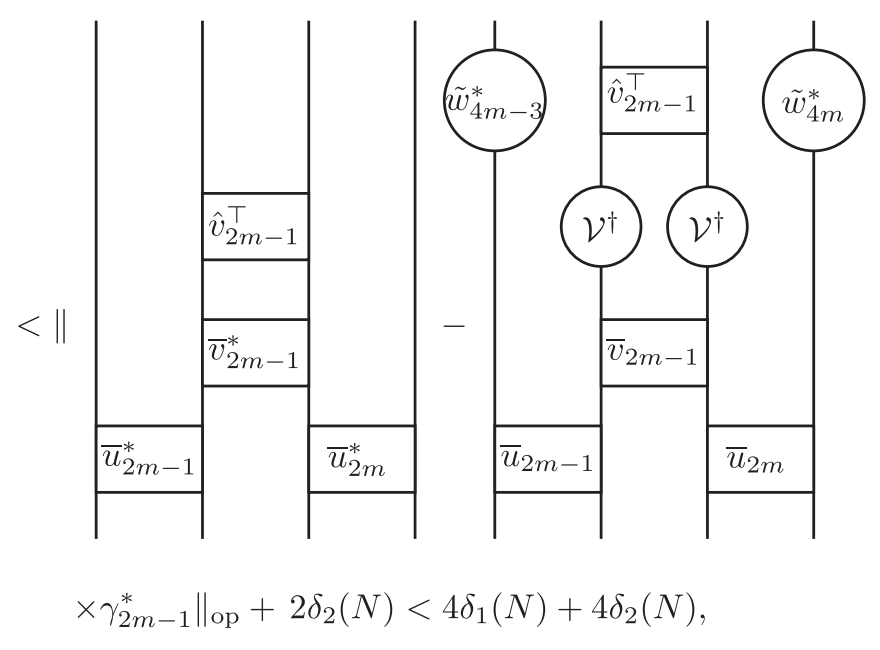

where we employed $\mathcal{V}^{\dagger}= \pm \mathcal{V}^{*}$, Eqs. (52) and (53) (each one twice) and $\left|\gamma_{k}\right| \rightarrow 1$ for $N \rightarrow \infty$. Using the triangular inequality and Eqs. (144) and (145), we thus arrive at

$$
\begin{aligned}
& \| \tilde{W}_{2 m-1}^{*} \otimes \tilde{W}_{2 m}^{*} \\
& \quad-\frac{\gamma_{2 m-1}^{*}}{\hat{\gamma}_{2 m-1}^{*}}\left(\tilde{w}_{4 m-3}^{*} \otimes \hat{w}_{4 m-2}^{\dagger}\right) \otimes\left(\hat{w}_{4 m-1}^{\dagger} \otimes \tilde{w}_{4 m}^{*}\right) \|_{\mathrm{op}} \\
& \quad<6 \delta_{1}(N)+4 \delta_{2}(N) .
\end{aligned}
$$

Hence,

$$
\left\|\tilde{W}_{2 m}^{*}-\kappa_{2 m} \hat{w}_{4 m-1}^{\dagger} \otimes \tilde{w}_{4 m}^{*}\right\|_{\text {op }}<6 \delta_{1}(N)+4 \delta_{2}(N)
$$

with $\kappa_{2 m} \in \mathbb{C}$ and $\left|\kappa_{2 m}\right| \rightarrow 1$ for $N \rightarrow \infty$. Finally, via Eq. (76)

$$
\begin{aligned}
& \left\|\tilde{W}_{2 m} \tilde{W}_{2 m}^{*}-\left|\kappa_{2 m}\right|^{2}\left(\hat{w}_{4 m-1}^{\top} \otimes \tilde{w}_{4 m}\right)\left(\hat{w}_{4 m-1}^{\dagger} \otimes \tilde{w}_{4 m}^{*}\right)\right\|_{\mathrm{op}} \\
& \quad=\left\|\tilde{W}_{2 m} \tilde{W}_{2 m}^{*}-\left|\kappa_{2 m}\right|^{2}\left(\hat{w}_{4 m-1}^{\top} \hat{w}_{4 m-1}^{\dagger}\right) \otimes\left(\tilde{w}_{4 m} \tilde{w}_{4 m}^{*}\right)\right\|_{\mathrm{op}} \\
& \quad<12 \delta_{1}(N)+8 \delta_{2}(N) .
\end{aligned}
$$


Owing to Eq. (141),

$$
\left\|\left[\bar{U}_{21}\right]_{: l}-\left[P^{\dagger}\left(\lambda_{2}\right) P\left(\lambda_{1}\right)\right]_{: l}\right\|_{2}<2 \delta_{1}(N)+O(\epsilon),
$$

where $[\cdots]_{: l}$ refers to the $l$ th column vector. In the limit $\epsilon \rightarrow 0$, the column vectors of $\bar{U}_{21}$ thus converge to the ones of $P^{\dagger}\left(\lambda_{2}\right) P\left(\lambda_{1}\right)$ with increasing $N$, which are product states. Hence, the topological index of $\bar{U}_{21}$ can only be +1 , since otherwise one could use Lemma 4 to show that the column vectors of $\bar{U}_{21}$ have approximately twofold degenerate entanglement spectra, which is not the case for product states. We thus have

$$
\left\|\mathbb{1}-\tilde{W}_{k} \tilde{W}_{k}^{*}\right\|_{\text {op }}<22 \delta_{1}(N)
$$

i.e., according to Eqs. (148) and (76)

$$
\begin{aligned}
\mid \mathbb{1} & -\left|\kappa_{2 m}\right|^{2}\left(\hat{w}_{4 m-1}^{\top} \hat{w}_{4 m-1}^{\dagger}\right) \otimes\left(\tilde{w}_{4 m} \tilde{w}_{4 m}^{*}\right) \|_{\mathrm{op}} \\
& <34 \delta_{1}(N)+8 \delta_{2}(N) .
\end{aligned}
$$

According to Lemma $3,\left\|\mathbb{1} \mp \tilde{w}_{j} \tilde{w}_{j}^{*}\right\|<11 \delta_{1}(N)$ and $\| \mathbb{1} \mp$ $\hat{w}_{j} \hat{w}_{j}^{*} \|<11 \delta_{1}(N)$. Therefore, for sufficiently large $N$, the topological indices of $\bar{U}\left(\lambda_{1}\right)$ and $\bar{U}\left(\lambda_{2}\right)$ have to be identical. In the thermodynamic limit, the topological index is thus conserved along the path.

As a corollary, we obtain that it is impossible to adiabatically move the system from the topologically nontrivial to the topologically trivial phase without breaking time reversal symmetry or violating the FMBL condition.

\section{CONCLUSIONS}

We proved the existence of an SPT phase of all eigenstates of FMBL systems invariant under time reversal symmetry. Using a two-layer quantum circuit, we demonstrated the four-fold degeneracy of the entanglement spectra of all eigenstates in the SPT phase. The obtained classification thus resembles the one of one-dimensional gapped ground states with time reversal symmetry $[84,85]$. An extension to on-site symmetries and fermionic systems is expected to yield similar results, but cannot be carried out with the tools introduced in this paper only, which crucially require the absorption of the phase matrix $\Theta$ into the tensor network.

We proved the robustness of the two phases to arbitrary symmetry preserving perturbations so long as the system remains FMBL. Thus, our results imply that there can be no symmetry-preserving transition between the topological and the trivial FMBL phase without the delocalization of at least some eigenstate(s), i.e., the system has to enter a critical regime. This behavior resembles SPT ground states in one dimension, which retain their topological properties unless the perturbations break the symmetry or close the energy gap leading to a nonlocal change of the ground state wave function. Note that this by no means implies that the above transition has similar features as the MBL-to-thermal transition [80]. In particular, there might be a crossover regime, where as a function of energy some eigenstates are localized and topologically trivial, whereas others are localized and SPT, with delocalized eigenstates separating those energy windows [82].

Similar approaches might be employed to fully classify symmetry-protected FMBL phases in one dimension. This would involve showing that there are no topological subclasses compared to the ones obtained for one-dimensional ground states, which was also not carried out in the current analysis. Note on the other hand that MBL systems cannot have nonAbelian symmetries [92].

Furthermore, the defined topological index might be used in numerical simulations with quantum circuits in order to map out the phase diagrams of MBL systems with time reversal symmetry. The advantage over exact diagonalization would be that the tensor network approach does not require prior knowledge of an order parameter [80,81] or the splitting between ideally degenerate energy levels for open boundary conditions to be smaller than the mean level spacing [78].

In two dimensions, even strongly disordered systems are believed to eventually equilibrate [96-98], though possibly on astronomically long time scales [98]. On short time scales, they behave many-body localized [21,22], and are thus well described by shallow two-dimensional quantum circuits [99]. A similar approach might therefore be used to prove short-time symmetry and localization protection in two dimensions.

\section{ACKNOWLEDGMENTS}

The author would like to thank Steven Simon for discussions and providing valuable feedback to an earlier manuscript. The author is also grateful to Christoph Sünderhauf, Norbert Schuch, Arijeet Pal, Amos Chan, Andrea De Luca, and David Pérez-García for helpful discussions. This work was supported by TOPNES, EPSRC Grant No. EP/I031014/1 and the European Commission under the Marie Curie Programme. The contents of this article reflect only the author's views and not the views of the European Commission. Statement of compliance with EPSRC policy framework on research data: This publication is theoretical work that does not require supporting research data.

\section{APPENDIX: CLUSTER STATE WITH RANDOM COUPLINGS}

If we set $\sigma_{h}=\sigma_{V}=0$ in the Hamiltonian (1), i.e.,

$$
H=\sum_{i=1}^{N} \lambda_{i} \sigma_{x}^{i-1} \sigma_{z}^{i} \sigma_{x}^{i+1},
$$

it is a sum of commuting local projectors (stabilizer code). As a result, the unitary matrix $U_{\mathrm{cl}}$ which diagonalizes the Hamiltonian can be written exactly as a two-layer quantum circuit [94] with $\ell=2$ (assuming $N$ to be even) and

$$
u_{k}=v_{k}=u_{\mathrm{cl}}=\frac{1}{2}\left(\begin{array}{rrrr}
1 & -1 & -1 & -1 \\
-1 & 1 & -1 & -1 \\
-1 & -1 & 1 & -1 \\
-1 & -1 & -1 & 1
\end{array}\right)
$$

In the $| \pm\rangle=\frac{1}{\sqrt{2}}(|0\rangle \pm|1\rangle)$ basis, $u_{\mathrm{cl}}$ is diagonal, i.e., all $u_{k}$ and $v_{k}$ extended by identities to the full Hilbert space of $N$ spins commute with each other. Note also that if we set $v_{N / 2}=\mathbb{1}$, the obtained quantum circuit diagonalizes the Hamiltonian with open boundary conditions, i.e., where the sum in Eq. (A1) extends only from 2 to $N-1$. 
One can easily verify that $u_{\mathrm{cl}}$ fulfills (setting $X:=\sigma_{x}, Z:=$ $\left.\sigma_{z}, I:=\mathbb{1}_{2 \times 2}\right)$

$$
\begin{aligned}
u_{\mathrm{cl}} & =-(I \otimes Z) u_{\mathrm{cl}}(X \otimes Z) \\
& =-(Z \otimes X) u_{\mathrm{cl}}(Z \otimes I) \\
& =-(Z \otimes I) u_{\mathrm{cl}}(Z \otimes X) \\
& =-(X \otimes Z) u_{\mathrm{cl}}(I \otimes Z) \\
& =-(Z \otimes Z) u_{\mathrm{cl}}[(X Z) \otimes(X Z)] \\
& =-[(X Z) \otimes(X Z)] u_{\mathrm{cl}}(Z \otimes Z),
\end{aligned}
$$

where Eq. (A7) follows from Eqs. (A3) and (A5) and Eq. (A8) from Eqs. (A4) and (A6). The symmetries also reflect the fact that the Hamiltonian commutes with $Z_{\text {even }}:=(I \otimes Z)^{\otimes \frac{N}{2}}$ and $Z_{\text {odd }}:=(Z \otimes I)^{\otimes \frac{N}{2}}$. Equations (A5) and (A6) yield

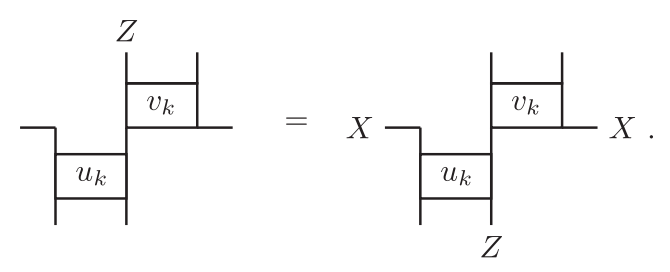

For periodic boundary conditions this implies $Z_{\mathrm{even}}\left|\psi_{l_{1} l_{2} \cdots l_{N}}^{\mathrm{PBC}}\right\rangle=$ $(-1)^{l_{2}+l_{4}+\cdots+l_{N}}\left|\psi_{l_{1} l_{2} \cdots l_{N}}^{\mathrm{PBC}}\right\rangle$, where $\left|\psi_{l_{1} l_{2} \cdots l_{N}}^{\mathrm{PBC}}\right\rangle$ is the column vector corresponding to the l-bit configuration $l_{1}, l_{2}, \ldots, l_{N}$ of $U_{\mathrm{cl}}^{\mathrm{PBC}}$ $\left(l_{i}=0,1\right)$. Similarly, Eqs. (A3) and (A4) imply

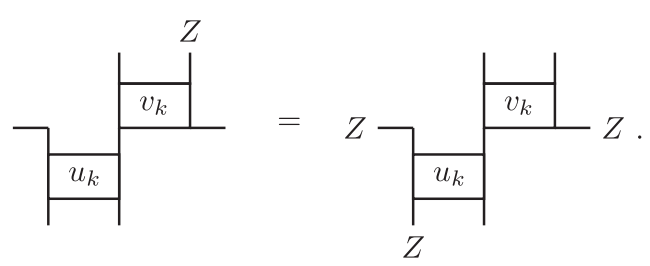

Therefore, we have $Z_{\text {odd }}\left|\psi_{l_{1} l_{2} \cdots l_{N}}^{\mathrm{PBC}}\right\rangle=(-1)^{l_{1}+l_{3}+\cdots+l_{N-1}}$ $\left|\psi_{l_{1} l_{2} \cdots l_{N}}^{\mathrm{PBC}}\right\rangle$ and as a result also $\sigma_{z}^{\otimes N}\left|\psi_{l_{1} l_{2} \cdots l_{N}}^{\mathrm{PBC}}\right\rangle=$ $(-1)^{\sum_{i=1}^{N} l_{i}}\left|\psi_{l_{1} l_{2} \cdots l_{N}}^{\mathrm{PBC}}\right\rangle$. This can be seen directly from the relation

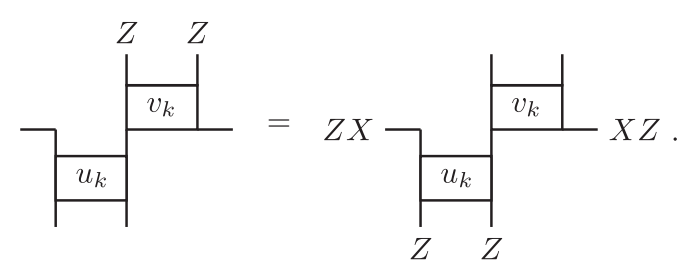

(A11)

which is obtained using Eqs. (A7) and (A8). This corresponds to the virtual symmetry $w_{2 k-1}=Z X$ and thus $w_{j} w_{j}^{*}=-\mathbb{1}$. Note that strictly speaking Eq. (A11) is not the same as Eq. (13) in the main part, since here we have a $Z \otimes Z$ operator acting on the 1-bit basis (i.e., $\Theta=\sigma_{z}^{\otimes N}$ ). These additional signs can be removed by defining the quantum circuit in terms of the unitaries $\left.\bar{u}_{k}=u_{\mathrm{cl}}\left[\begin{array}{cc}1 & 0 \\ 0 & i\end{array}\right) \otimes\left(\begin{array}{ll}1 & 0 \\ 0 & i\end{array}\right)\right]$ and $\bar{v}_{k}=u_{\mathrm{cl}}$. This comes at the price of making $\bar{u}_{k}$ complex [such that the complex conjugate appears on the left hand side of Eq. (A11) in accordance with Eq. (13)]. The corresponding overall unitary matrix $\bar{U}_{\mathrm{cl}}$ still diagonalizes the Hamiltonian and we still have $\bar{w}_{j} \bar{w}_{j}^{*}=-\mathbb{1}$.
As an add-on, let us remark that Eqs. (A3) to (A8) also indicate how degenerate eigenstates for open boundary conditions are related by 1-bit flips at the edges. The degeneracy arises because $\left|\psi_{l_{1} \ldots l_{N}}^{\mathrm{OBC}}\right\rangle, Z_{\text {even }}\left|\psi_{l_{1} \ldots l_{N}}^{\mathrm{OBC}}\right\rangle, Z_{\text {odd }}\left|\psi_{l_{1} \cdots l_{N}}^{\mathrm{OBC}}\right\rangle$ and $\sigma_{z}^{\otimes N}\left|\psi_{l_{1} \ldots l_{N}}^{\mathrm{OBC}}\right\rangle$ have the same energy (since $Z_{\text {even }}$ and $Z_{\text {odd }}$ commute with the Hamiltonian). As we will see, those states are linearly independent, i.e., the energy spectrum is fourfold degenerate. Noting that $v_{N / 2}=\mathbb{1}$, we analyze the symmetries

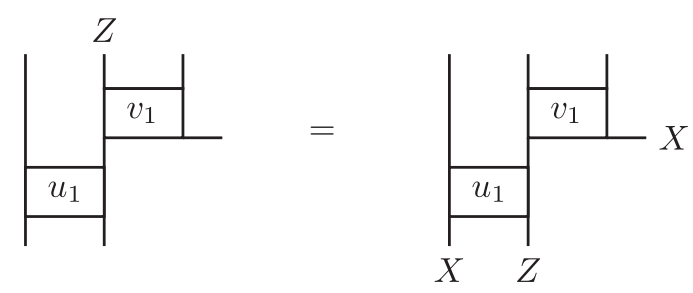

[obtained by using Eqs. (A5) and (A3)] and

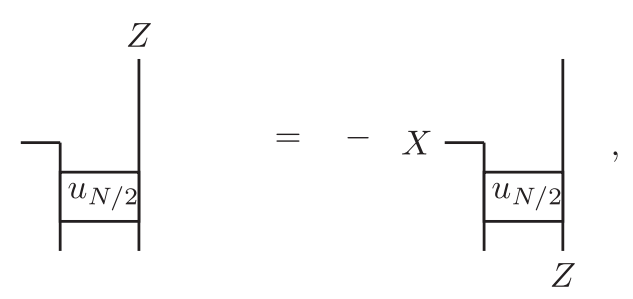

which follows from Eq. (A6). Using these relations along with Eq. (A9), we find $Z_{\text {even }}\left|\psi_{l_{1} l_{2} \cdots l_{N}}^{\mathrm{OBC}}\right\rangle=(-1)^{1+l_{2}+l_{4}+\cdots+l_{N}}\left|\psi_{\bar{l}_{1} l_{2} \cdots l_{N}}^{\mathrm{OBC}}\right\rangle$ with $\bar{l}_{i}:=1-l_{i}$. Similarly we obtain

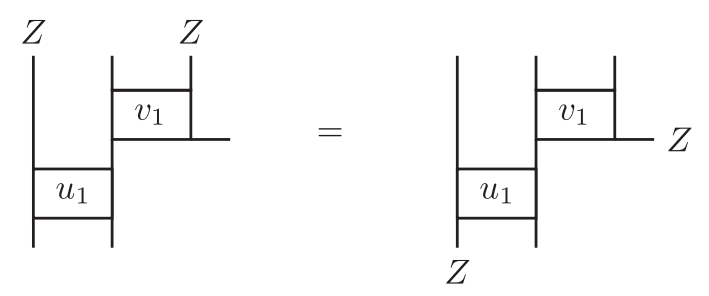

from Eqs. (A3) and (A4) and

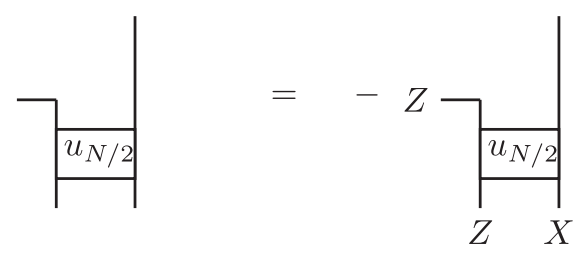

from Eq. (A5). The last two equations combined with Eq. (A10) tell us that $Z_{\text {odd }}\left|\psi_{l_{1} \cdots l_{N-1} l_{N}}^{\mathrm{OBC}}\right\rangle=$ $(-1)^{1+l_{1}+l_{3}+\cdots+l_{N-1}}\left|\psi_{l_{1} \cdots l_{N-1} \bar{I}_{N}}^{\mathrm{OBC}}\right\rangle$. As a result, we have $\sigma_{z}^{\otimes N}\left|\psi_{l_{1} l_{2} \cdots l_{N-1} l_{N}}^{\mathrm{OBC}}\right\rangle=(-1)^{1+\sum_{i=1}^{N} l_{i}}\left|\psi_{\bar{l}_{1} l_{2} \cdots l_{N-1} \bar{l}_{N}}^{\mathrm{OOCC}}\right\rangle$. 
[1] P. Anderson, Phys. Rev. 109, 1492 (1958).

[2] P. A. Lee and T. V. Ramakrishnan, Rev. Mod. Phys. 57, 287 (1985).

[3] L. Fleishman and P. W. Anderson, Phys. Rev. B 21, 2366 (1980).

[4] I. V. Gornyi, A. D. Mirlin, and D. G. Polyakov, Phys. Rev. Lett. 95, 206603 (2005).

[5] D. M. Basko, I. L. Aleiner, and B. L. Altshuler, Ann. Phys. 321, 1126 (2006).

[6] J. Z. Imbrie, J. Stat. Phys. 163, 998 (2016).

[7] R. Nandkishore and D. A. Huse, Annu. Rev. Condens. Matter Phys. 6, 15 (2015).

[8] E. Altman and R. Vosk, Annu. Rev. Condens. Matter Phys. 6, 383 (2015).

[9] D. J. Luitz and Y. B. Lev, Ann. Phys. 529, 1600350 (2017).

[10] J. Z. Imbrie, V. Ros, and A. Scardicchio, Ann. Phys. 529, 1600278 (2017).

[11] D. A. Abanin and Z. Papić, Ann. Phys. 529, 1700169 (2017).

[12] F. Alet and N. Laflorencie, C. R. Physique (2018), doi: 10.1016/j.crhy.2018.03.003.

[13] A. Peres, Phys. Rev. A 30, 504 (1984).

[14] J. M. Deutsch, Phys. Rev. A 43, 2046 (1991).

[15] M. Srednicki, Phys. Rev. E 50, 888 (1994).

[16] M. Srednicki, J. Phys. A 32, 1163 (1999).

[17] M. Rigol, V. Dunjko, and M. Olshanii, Nature (London) 452, 854 (2008).

[18] L. D'Alessio, Y. Kafri, A. Polkovnikov, and M. Rigol, Adv. Phys. 65, 239 (2016).

[19] F. Borgonovi, F. Izrailev, L. Santos, and V. Zelevinsky, Phys. Rep. 626, 1 (2016).

[20] M. Schreiber, S. S. Hodgman, P. Bordia, H. P. Lüschen, M. H. Fischer, R. Vosk, E. Altman, U. Schneider, and I. Bloch, Science 349, 842 (2015).

[21] J.-y. Choi, S. Hild, J. Zeiher, P. Schauß, A. Rubio-Abadal, T. Yefsah, V. Khemani, D. A. Huse, I. Bloch, and C. Gross, Science 352, 1547 (2016).

[22] P. Bordia, H. Lüschen, S. Scherg, S. Gopalakrishnan, M. Knap, U. Schneider, and I. Bloch, Phys. Rev. X 7, 041047 (2017).

[23] G. D. Chiara, S. Montangero, P. Calabrese, and R. Fazio, J. Stat. Mech. (2006) P03001.

[24] J. H. Bardarson, F. Pollmann, and J. E. Moore, Phys. Rev. Lett. 109, 017202 (2012).

[25] M. Serbyn, Z. Papić, and D. A. Abanin, Phys. Rev. Let. 110, 260601 (2013).

[26] D. J. Luitz, N. Laflorencie, and F. Alet, Phys. Rev. B 93, 060201 (2016).

[27] R. Singh, J. H. Bardarson, and F. Pollmann, New J. Phys. 18, 023046 (2016).

[28] T. Zhou and D. J. Luitz, Phys. Rev. B 95, 094206 (2017).

[29] V. Oganesyan and D. A. Huse, Phys. Rev. B 75, 155111 (2007).

[30] T. C. Berkelbach and D. R. Reichman, Phys. Rev. B 81, 224429 (2010).

[31] A. Pal and D. A. Huse, Phys. Rev. B 82, 174411 (2010).

[32] D. Pekker, G. Refael, E. Altman, E. Demler, and V. Oganesyan, Phys. Rev. X 4, 011052 (2014).

[33] R. Vosk and E. Altman, Phys. Rev. Lett. 112, 217204 (2014).

[34] R. Vosk, D. A. Huse, and E. Altman, Phys. Rev. X 5, 031032 (2015).

[35] A. C. Potter, R. Vasseur, and S. A. Parameswaran, Phys. Rev. X 5, 031033 (2015).
[36] D. J. Luitz, N. Laflorencie, and F. Alet, Phys. Rev. B 91, 081103 (2015).

[37] T. Devakul and R. R. P. Singh, Phys. Rev. Lett. 115, 187201 (2015).

[38] S. Gopalakrishnan, K. Agarwal, E. A. Demler, D. A. Huse, and M. Knap, Phys. Rev. B 93, 134206 (2016).

[39] M. Žnidarič, A. Scardicchio, and V. K. Varma, Phys. Rev. Lett. 117, 040601 (2016).

[40] S. L. Sondhi, S. M. Girvin, J. P. Carini, and D. Shahar, Rev. Mod. Phys. 69, 315 (1997).

[41] X. Yu, D. J. Luitz, and B. K. Clark, Phys. Rev. B 94, 184202 (2016).

[42] L. Zhang, B. Zhao, T. Devakul, and D. A. Huse, Phys. Rev. B 93, 224201 (2016).

[43] K. Agarwal, E. Altman, E. Demler, S. Gopalakrishnan, D. A. Huse, and M. Knap, Ann. Phys. 529, 1600326 (2017).

[44] V. Khemani, S. P. Lim, D. N. Sheng, and D. A. Huse, Phys. Rev. X 7, 021013 (2017).

[45] S. A. Parameswaran, A. C. Potter, and R. Vasseur, Ann. Phys. 529, 1600302 (2017).

[46] V. Khemani, D. N. Sheng, and D. A. Huse, Phys. Rev. Lett. 119, 075702 (2017).

[47] S. Sachdev, Quantum Phase Transitions (Wiley Online Library, New York, 2007).

[48] M. Serbyn, Z. Papić, and D. A. Abanin, Phys. Rev. Lett. 111, 127201 (2013).

[49] A. Chandran, I. H. Kim, G. Vidal, and D. A. Abanin, Phys. Rev. B 91, 085425 (2015).

[50] V. Ros, M. Mueller, and A. Scardicchio, Nucl. Phys. B 891, 420 (2015).

[51] S. Inglis and L. Pollet, Phys. Rev. Lett. 117, 120402 (2016).

[52] L. Rademaker and M. Ortuño, Phys. Rev. Lett. 116, 010404 (2016).

[53] C. Monthus, J. Stat. Mech. (2016) 033101.

[54] D. Pekker, B. K. Clark, V. Oganesyan, and G. Refael, Phys. Rev. Lett. 119, 075701 (2017).

[55] M. Goihl, M. Gluza, C. Krumnow, and J. Eisert, Phys. Rev. B 97, 134202 (2018).

[56] A. K. Kulshreshtha, A. Pal, T. B. Wahl, and S. H. Simon, arXiv:1707.05362.

[57] S. D. Geraedts, R. N. Bhatt, and R. Nandkishore, Phys. Rev. B 95, 064204 (2017).

[58] C. Monthus, J. Phys. A: Math. Theor. 51, 195301 (2018).

[59] B. Bauer and C. Nayak, J. Stat. Mech. (2013) P09005.

[60] A. Pal, Ph.D. thesis, Princeton University, 2012.

[61] M. Friesdorf, A. H. Werner, W. Brown, V. B. Scholz, and J. Eisert, Phys. Rev. Lett. 114, 170505 (2015).

[62] M. B. Hastings and T. Koma, Commun. Math. Phys. 265, 781 (2006).

[63] D. Pérez-García, F. Verstraete, M. M. Wolf, and J. I. Cirac, Quantum Inf. Comput. 7, 401 (2007).

[64] M.Žnidarič, T. Prosen, and P. Prelovšek, Phys. Rev. B 77, 064426 (2008).

[65] V. Khemani, F. Pollmann, and S. L. Sondhi, Phys. Rev. Lett. 116, 247204 (2016).

[66] D. M. Kennes and C. Karrasch, Phys. Rev. B 93, 245129 (2016).

[67] S. P. Lim and D. N. Sheng, Phys. Rev. B 94, 045111 (2016).

[68] X. Yu, D. Pekker, and B. K. Clark, Phys. Rev. Lett. 118, 017201 (2017). 
[69] T. Devakul, V. Khemani, F. Pollmann, D. A. Huse, and S. L. Sondhi, Philos. Trans. R. Soc. London Sect. A 375, 20160431 (2017).

[70] F. Verstraete and J. I. Cirac, arXiv:cond-mat/0407066.

[71] J. I. Cirac and F. Verstrate, J. Phys. A 42, 504004 (2009).

[72] J. Eisert, Model. Simul. 3, 520 (2013).

[73] R. Orús, Ann. Phys. 349, 117 (2014).

[74] D. Pekker and B. K. Clark, Phys. Rev. B 95, 035116 (2017).

[75] A. Chandran, J. Carrasquilla, I. H. Kim, D. A. Abanin, and G. Vidal, Phys. Rev. B 92, 024201 (2015).

[76] F. Pollmann, V. Khemani, J. I. Cirac, and S. L. Sondhi, Phys. Rev. B 94, 041116 (2016).

[77] T. B. Wahl, A. Pal, and S. H. Simon, Phys. Rev. X 7, 021018 (2017).

[78] D. A. Huse, R. Nandkishore, V. Oganesyan, A. Pal, and S. L. Sondhi, Phys. Rev. B 88, 014206 (2013).

[79] A. Chandran, V. Khemani, C. R. Laumann, and S. L. Sondhi, Phys. Rev. B 89, 144201 (2014).

[80] J. A. Kjäll, J. H. Bardarson, and F. Pollmann, Phys. Rev. Lett. 113, 107204 (2014).

[81] Y. Bahri, R. Vosk, E. Altman, and A. Vishwanath, Nat. Commun. 6, 7341 (2015).

[82] K. Slagle, Z. Bi, Y.-Z. You, and C. Xu, arXiv:1505.05147.

[83] A. C. Potter and A. Vishwanath, arXiv:1506.00592.

[84] F. Pollmann, A. M. Turner, E. Berg, and M. Oshikawa, Phys. Rev. B 81, 064439 (2010).
[85] X. Chen, Z.-C. Gu, and X.-G. Wen, Phys. Rev. B 83, 035107 (2011).

[86] N. Schuch, D. Pérez-García, and I. Cirac, Phys. Rev. B 84, 165139 (2011).

[87] F. Verstraete and J. I. Cirac, Phys. Rev. B 73, 094423 (2006).

[88] Y. Huang, arXiv:1505.00772.

[89] D. A. Huse, R. Nandkishore, and V. Oganesyan, Phys. Rev. B 90, 174202 (2014).

[90] W. DeRoeck, F. Huveneers, M. Müller, and M. Schiulaz, Phys. Rev. B 93, 014203 (2016).

[91] M. Sanz, M. M. Wolf, D. Pérez-García, and J. I. Cirac, Phys. Rev. A 79, 042308 (2009).

[92] A. C. Potter and R. Vasseur, Phys. Rev. B 94, 224206 (2016).

[93] N. Bultinck, D. J. Williamson, J. Haegeman, and F. Verstraete, Phys. Rev. B 95, 075108 (2017).

[94] S. O. Skrøvseth and S. D. Bartlett, Phys. Rev. A 80, 022316 (2009).

[95] J. I. Cirac, D. Poilblanc, N. Schuch, and F. Verstraete, Phys. Rev. B 83, 245134 (2011).

[96] A. Chandran, A. Pal, C. R. Laumann, and A. Scardicchio, Phys. Rev. B 94, 144203 (2016).

[97] W. D. Roeck and J. Z. Imbrie, Philos. Trans. R. Soc. London Sect. A 375, 20160422 (2017).

[98] P. Ponte, C. R. Laumann, D. A. Huse, and A. Chandran, Philos. Trans. R. Soc. London Sect. A 375, 20160428 (2017).

[99] T. B. Wahl, A. Pal, and S. H. Simon, arXiv:1711.02678. 